Center for

Mathematical Economics

Working Papers

\title{
494
}

July 2013

\section{Explicit Collusion under Antitrust Enforcement}

Igor Mouraviev

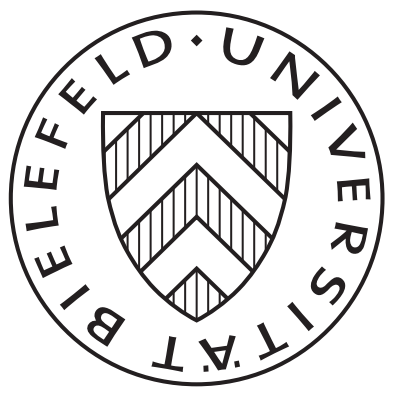

Center for Mathematical Economics (IMW) 


\title{
Explicit Collusion under Antitrust Enforcement*
}

\author{
Igor Mouraviev ${ }^{\dagger}$
}

July 14, 2013

\begin{abstract}
The article seeks to fill the gap between tacit and explicit collusion in a setting where firms observe only their own output levels and a common price, which includes a stochastic component. Without communication, firms fail to discriminate between random shocks and marginal deviations, which constrains the scope for collusion. By eliminating uncertainty about what has happened, communication facilitates detection of deviations but reduces collusive profits due to the risk of exposure to legal sanctions. With the optimal collusive strategy, firms communicate only if the market price falls somewhat below the trigger price. Moreover, they tend to communicate more often as they become less patient, a cartel grows in size, or demand uncertainty rises.
\end{abstract}

Keywords: Collusion, Communication, Imperfect Monitoring, Frequency of Meetings.

JEL Classification: D82, L41.

${ }^{*}$ I am especially grateful to Patrick Rey for much help and advise while preparing this article. I wish to acknowledge, without implicating in any way, detailed and valuable comments of Joe Harrington, Leslie Marx and Johan Stennek. I also thank participants at the EARIE Conference in Rome, the Meeting of Econometric Society in Melbourne, the Conference at the Mannheim Centre for Competition and Innovation, and International Industrial Organization Conference in Arlington for helpful discussions. Part of the paper was written while I was a faculty member at the Higher School of Economics.

${ }^{\dagger}$ Center for Mathematical Economics (IMW), Bielefeld University, muravievigor@gmail.com 


\section{Introduction}

The conventional economic theory posits that collusion can be supported by a Nash equilibrium in a game of repeated interaction, provided that each firm correctly anticipates the strategic response of its rivals. A distinct feature of the economic approach is that it rules out the need for any explicit agreement and instead emphasizes the importance of implicit understanding among firms to coordinate their behavior so as to achieve profits above competitive levels. By contrast, the law distinguishes collusive behavior only if it is supported by the evidence demonstrating that firms have concluded an agreement "which ha[s] as [its] object or effect the prevention, restriction or distortion of competition ..." ${ }^{1}$ Not surprisingly, in almost all cartel cases brought forward by the European Commission it has been found that firms systematically engaged in express communication, as evidenced by the revealed notes of cartel meetings made by the representatives of these firms.

In attempting to reduce the tension between legal and economic concepts of collusion, the extant literature has primarily focused on the question of why collusive firms communicate. By contrast, the objective of this paper is to study the question of when they will choose to do so. One reason for raising this issue is that sharing of commercially sensitive information (e.g., customer lists, actual prices, realized sales) commonly requires cartels to develop the means to monitor and verify the reports of their members. For example, the pre-insulated pipe cartel often turned to independent auditors to certify the accuracy of the data being exchanged. ${ }^{2}$ In much the same vein, the organic peroxides cartel used the service of AC Treuhand who "acted independently from the cartel members by undertaking and approving the auditing, which was an essential feature of the agreement." ${ }^{3}$ Apart from this, cartel meetings often leave traces of evidence of concerted efforts to restrict competition and, in such a way, they expose conspirators to the risk of legal prosecution. For these reasons, express communication is not only beneficial: it also makes collusion less profitable due to the costs associated with either antitrust enforcement or setting up and maintaining a monitoring scheme.

On the other hand, firms can always avoid paying these costs by colluding tacitly. For instance, many antitrust officials share the view that if they could achieve a collusive outcome without express communication, then it is unlikely that they would

\footnotetext{
${ }^{1}$ The quotation is part of Article 81 of the Treaty of Rome.

2 "Following the audit (carried out by Swiss accountants), which ascertained the figures for each producer's 1992 revenue (...), the producers met in Zurich on 18 or 19 August 1993 [...]" (Case No IV/35.691/E-4 - Pre-Insulated Pipe Cartel, §50)

${ }^{3}$ Case COMP/E-2/37.857 - Organic Peroxides, $§ 333$.
} 
ever be accused of wrongdoing because of the lack of incriminating evidence. ${ }^{4}$ This however raises the questions of whether collusive firms should ever use communication and, if they should, how they could minimize the costs associated with doing so.

To address these issues, the paper considers a setting similar to Green and Porter (1984), but modifies it by giving firms the opportunity to communicate. Specifically, in each period firms can meet and exchange reliable information about their past output levels before they engage in product market competition. To capture the stylized evidence, the model further assumes that cartel meetings can be uncovered by the competition authority, in which case firms face fines and damages claims. As in Green and Porter, the inability of firms to perfectly detect deviations from the collusive output constraints the scope for collusion. The key difference lies in the fact that they can now deter deviations not only by reverting to a temporary price war but also by verifying the exact amount of goods supplied to the market. Since both ways are expensive, firms face a nontrivial dilemma in choosing the one which maximizes expected profits.

The main result of the paper is that, with the uniform distribution of the demand shocks used here, the optimal collusive strategy has the following form: firms meet whenever the market price falls somewhat below a trigger price, and inflict maximal punishment as soon as they detect that some firm has either deviated from the collusive output or waived the meeting when it should be held. In contrast to Green and Porter, the strategy induces the pattern of collusive behavior in which firms never revert to a temporary punishment phase. Put it differently, they hold meetings, but the meetings just confirm the compliance with the collusive agreement. Intuitively, communication, in this setting, has an important deterrence effect: by increasing the likelihood of detection of deviations, it relaxes incentives to deviate. ${ }^{5}$ More precisely, given that any detected deviation is sanctioned by a price war, it allows firms to deter all potential deviations without involving any cost associated with the punishment phase.

Another distinct feature of the strategy obtained is that, whenever the market experiences a price decrease, firms convene to discuss what happens. This result is due to the uniform distribution of the demand shocks which, in particular, implies that the probability for the market price to fall within some range is invariant with respect to marginal changes in total output. In turn, given that the best deviation

\footnotetext{
${ }^{4}$ The common presumption is that the law must not prohibit "a rational calculation by each seller of what the consequences of his price decision would be, taking into account the probable or virtually certain reactions of his competitors". (Turner, 1962)

${ }^{5}$ To be more precise, given that the distribution of the demand shocks has a bounded support, some deviations can also be revealed by certain price realizations. This feature, however, does not diminish the value of communication and disappears when the variance of the demand shocks becomes sufficiently large.
} 
by a firm consists in increasing its output, this property implies that, by moving the range of prices triggering communication toward the lower end of the price spectrum, firms increase the probability of detection of deviations without changing the costs of communication. Consequently, if they ever contemplate communicating, then doing so is most optimal when the price falls somewhat below the trigger price.

There is also some evidence from antitrust practice supporting the predictions of the model. In the Sorbates Case, for example, the Decision of the European Commission states:

"Hoechst and the Japanese producers monitored target price adherence through the data regarding competitor pricing which they used to receive through their dealers. When prices fell below the target prices for key customers, the Japanese companies (mainly Daicel) and Hoechst did on occasions telephone each other to try to ensure that such prices were brought closer into line with the targets in the next large contract with the same customer." 6

The paper also sheds light on the determinants of the frequency of cartel meetings. Overall, the results suggest that conspirators are likely to meet more often as they become less patient, a cartel grows in size, or demand volatility rises. Intuitively, in all these environments they are more tempted to deviate from a cartel agreement, both because they may gain more from increasing their outputs and because they have less to fear that such deviations will be detected. As a result, in order to curb the temptation to deviate, cartels increase the intensity of monitoring.

The rest of the paper proceeds as follows. The next section provides a brief review of the relevant economic literature. Section 3 outlines the framework for the analysis. Section 4 derives the most profitable collusive strategy and characterizes the impact of communication on collusion. Section 5 studies the frequency of cartel meetings as a function of various factors that affect the sustainability of collusion. Concluding remarks are given in Section 6.

\section{Literature Review}

Since the time when the traditional structure-conduct-performance paradigm gained widespread acceptance, it has been recognized that firms communicate because they need to reach an agreement and coordinate on a collusive outcome. In their study of the Sugar Institute Case, Genesove and Mullin (2001) bring new insights to the role

\footnotetext{
${ }^{6}$ Case COMP/E-1/37.370 - Sorbates, $§ 113$.
} 
of communication in collusion. ${ }^{7}$ In particular, they argue that communication can also enhance the efficiency and the sustainability of collusion. The recent theoretical contributions demonstrate how it can serve these purposes.

The paper by Athey and Bagwell (2001), for instance, touches upon the issue of efficiency. More precisely, it shows that, in order to achieve productive efficiency, firms exchange private information about their unit costs. By contrast, Compte (1998) and Kandori and Matsushima (1998) explore the issue of sustainability in a setting where each firm's actions are privately observable and each firm receives a private and imperfect signal about rivals' play. Both papers demonstrate that the exchange of private information can help sustain collusion, provided that firms are sufficiently patient. ${ }^{8}$ In a related study, Harrington and Skrzypacz (2011) address a similar question but in a setting where firms hold private information about their prices and sales. Assuming that monetary transfers are possible, the authors show that communication constitutes an integral part of the mechanism that ensures the proper functioning of a cartel.

The current paper differs from the literature in two respects. First, in all the aforementioned papers it is assumed that communication is costless and the main issue is how to induce truthful reporting. In contrast, in my model firms cannot distort the reports but rather bear the costs of communication. Accordingly, the emphasis is placed not on truthful reporting (which is guaranteed by the assumption), but on the most efficient use of the limited information.

Second, the paper also differs in the way collusion is uncovered by the competition authorities. The literature typically treats the incriminating evidence as a "black box" or rather it assumes that collusion can be inferred from price observations. Either approach seems of little relevance for policy implications. ${ }^{9}$ In contrast, my model builds on the idea that the revealed evidence of communication is the main proof of collusive behavior. Consequently, in such a setting monitoring of prices is sensible insofar it helps to predict when collusive firms are more likely to communicate.

The closest paper to mine is perhaps Martin (2006) who pursues a somewhat similar approach. There are however two important differences. In his model, collusion with communication is an assumption while, in mine, it is derived as part of the

\footnotetext{
${ }^{7}$ See also Kuhn (2001) for the detailed discussion of how collusive firms can benefit from a particular type of information exchange.

${ }^{8}$ Although both papers show that the collusive scheme can involve communication, one question remains unsolved. As the authors themselves acknowledge, they fail to characterize the collusive scheme without communication and, as such, evaluate the benefits of communication.

${ }^{9}$ The Wood Pulp Case (OJL 85/1, 1985) clearly illustrates the point. In this case the Commission brought forward the enormous evidence of almost simultaneous price increases but failed to provide the evidence of explicit cooperation. Thus, much of the decision hinged upon the interpretation of the parallel behavior. While the Commission tried to prove that it was a consequence of collusion, the court did not accept its arguments on the grounds that it was equally consistent with independent and individually rational responses of firms in the market environment.
} 
optimal collusive strategy. Additionally, in contrast to my approach, he assumes that competition enforcement relies on price observations while abstracting from the nature of the evidence of collusion.

\section{Framework}

Consider a setting in which $n \geq 2$ identical firms produce a homogeneous good at fixed cost $k>0$ and constant marginal cost which is normalized to zero, without loss of generality. Firms engage in repeated Cournot competition over an infinite time horizon $t \in\{0,1, .$.$\} . Specifically, in period t$ firm $i$ chooses the quantity $q_{i t}$ to supply to the market. Given the total quantity supplied $Q_{t}=\sum_{i=1}^{n} q_{i t}$, the market clears at the price:

$$
p_{t}\left(Q_{t}\right)=\max \left\{0, a+\varepsilon_{t}-Q_{t}\right\},
$$

where $\varepsilon_{t}$ is the demand shock realized in period $t$ and $a>0$. The shocks are independent across the time and uniformly distributed over the interval $[-\Delta, \Delta]$. The parameter $\Delta$ measures the degree of demand uncertainty and is such that $0<\Delta<a /(n+1)$. The latter inequality implies that in competitive equilibrium firms face a positive market price even if the lowest-demand state is realized.

For a given profile of quantities supplied $\left(q_{1 t}, . ., q_{n t}\right)$, firm $i$ obtains the expected profit: ${ }^{10}$

$$
\pi_{i}^{e}\left(q_{1 t}, . ., q_{n t}\right)=\left(a-\Sigma_{j=1}^{n} q_{j t}\right) q_{i t}-k .
$$

If firms could coordinate their decisions so as to maximize the total industry profit, then each of them would produce the quantity $q^{m}=\arg \max _{q} \pi_{i}^{e}(q, . ., q)=a / 2 n$ and earn the expected profit $\Pi^{m}=n\left(q^{m}\right)^{2}-k$. In contrast, in the static Nash equilibrium each firm would produce the quantity $q^{n}=a /(n+1)$ and earn the expected profit $\pi^{n}=\left(q^{n}\right)^{2}-k$.

Following the approach of Green and Porter (1984), firms are assumed to observe only their own output levels and realized market prices. Since the main obstacle for collusion in this setting is the lack of perfect knowledge about each other's behavior, then firms could only facilitate collusion by exchanging private information about the exact quantities supplied. ${ }^{11}$ However, as was discussed in the Introduction, the costs associated with exchanging such information prevent them from systematically doing so. For the sake of exposition, it will be assumed that the costs are due to antitrust

\footnotetext{
${ }^{10}$ Hereinafter, it will be used repeatedly that the expected value of $\varepsilon$ is zero.

${ }^{11}$ If firms could manipulate the data, then there would be no scope for meaningful communication. This is because the information being exchanged refers to the past and, as such, it cannot affect firms' current and future profits. Consequently, little can be done to elicit it truthfully.
} 
enforcement. More precisely, it will be assumed that in every period the competition authority chooses an industry at random and conducts an audit on a regular basis. In case it finds that firms have met and exchanged information about the individual output levels, it levies fines against each firm participated in the meeting. ${ }^{12}$

To formalize matters, denote by $\mathcal{D} \equiv\{M, N\}$ the set of decision variables (which is common to all firms) where $M$ means that a firm proposes to meet and $N$ means that it does not do so. Also, denote by $\mathcal{P}=[0, a+\Delta]$ the set of feasible price realizations and by $\mathcal{Q}=[0, \bar{Q}]$ the set of feasible output levels where $\bar{Q} \geq a+\Delta$.

Repeated interaction between firms is modeled as an infinitely repeated game $G^{\infty}(\delta)$ defined by the component game $G$ and the common discount factor $\delta \in(0,1)$. The one-period game $G$ in turn is defined as follows:

Stage 1. Each firm $i$ makes a decision $d_{i} \in \mathcal{D}$. After all the decisions having been made, they become commonly known. The meeting takes place only if all firm agree on this, i.e., only if $d_{i}=M$ for all $i$. In which case, they disclose private information about the exact outputs produced in the past. If the meeting does not take place, neither firm can access private information about the rivals' output levels.

Stage 2. Each firm $i$ produces an output $q_{i} \in \mathcal{Q}$; the shock $\varepsilon$ is realized, and the market price $p \in \mathcal{P}$ becomes publicly observable.

Stage 3. The competition authority audits the industry. If the meeting has taken place, it detects the meeting with the probability $\rho \in(0,1)$ and levies the fixed fine $F$ against each firm attended the meeting. ${ }^{13}$ Otherwise, it finds no evidence of collusion and levies no fines.

Stage 4. The profits are obtained.

The timing of the game $G$ thus implies that, upon entering every single period, each firm observes the history of its own output levels, the realized market prices, the rivals' decisions regarding the meetings and the rivals' output levels disclosed during the meetings. Denote by $\mathcal{I} \equiv\{\varnothing\} \cup \mathcal{Q}^{n-1}$ the information set available to each firm in each period $t \geq 1$ which represents its knowledge of the rivals' output levels produced in period $t-1$; the sign $\varnothing$ means that a firm lacks any relevant information. ${ }^{14}$ Denote also by $\mathcal{H}_{t} \equiv \mathcal{Q}^{t-1} \times \mathcal{D}^{n(t-1)} \times \mathcal{I}^{t-1} \times \mathcal{P}^{t-1}$ the history up to date $t \geq 1$ and by $\mathcal{H}_{0}$ the null history. A strategy $\sigma_{i}$ for each firm $i$ specifies a sequence

\footnotetext{
${ }^{12}$ The model thus abstracts from any reason to communicate other than facilitate collusion.

${ }^{13}$ In general, the fine $F$ can vary with the total output $Q$. For example, it can be proportional to the damage caused to consumers. Alternatively, it can be associated with the foregone profits from the breakdown of collusion for a number of periods. The implicit assumption here is that firms neglect the relationship between $Q$ and $F$ because, say, it might be difficult to predict the judgement of court. An explicit account for a functional relationship between $Q$ and $F$ is the subject for future research.

${ }^{14}$ Note that $\mathcal{I} \equiv\{\varnothing\}$ at $t=0$ since there is nothing to disclose yet.
} 
of pair functions $\sigma_{i t} \equiv\left(d_{i t}, q_{i t}\right)$ defined for each period $t$ such that $d_{i t}: \mathcal{H}_{t} \longrightarrow \mathcal{D}$ and $q_{i t}: \mathcal{H}_{t} \times \mathcal{D}^{n} \longrightarrow \mathcal{Q}$. A strategy profile $\boldsymbol{\sigma}$ comprises the strategies of all firms and writes as $\boldsymbol{\sigma}=\left(\sigma_{1}, \ldots, \sigma_{n}\right)$. For a given $\boldsymbol{\sigma}$, firm $i$ obtains the expected payoff: ${ }^{15}$

$$
V_{i}\left(\sigma_{i}, \sigma_{-i}\right)=(1-\delta) \sum_{t=0}^{\infty} \delta^{t} v_{i}\left(\sigma_{i t}, \sigma_{-i t}\right),
$$

where $v_{i}\left(\sigma_{i t}, \sigma_{-i t}\right)=\pi_{i}^{e}\left(q_{1 t}, q_{-i t}\right)-\rho F$ if $d_{1 t}=. .=d_{n t}=M$ and $v_{i}\left(\sigma_{i t}, \sigma_{-i t}\right)=$ $\pi_{i}^{e}\left(q_{1 t}, q_{-i t}\right)$ otherwise.

Following the approach of Fudenberg, Levine, and Maskin (1994), the paper will consider those sequential equilibria in which in every single period firms condition their play on the history of the commonly observed variables only. Such strategies are called public strategies and such sequential equilibria are called perfect public equilibria. Furthermore, the analysis will focus on fully symmetric perfect public equilibria in which firms use the same continuation strategy, i.e., $\sigma_{i t}=\sigma_{t}$ for all $i$.

Denote by $\overline{\boldsymbol{\sigma}}=(\bar{\sigma}, \ldots, \bar{\sigma})$ (respectively, by $\underline{\boldsymbol{\sigma}}=(\underline{\sigma}, \ldots, \underline{\sigma})$ ) the equilibrium strategy profile which yields the maximal payoff $\bar{V}$ (respectively, the minimal payoff $\underline{V}$ ) in the game $G^{\infty}(\delta)$. Define the outcome path $\overline{\mathcal{Z}}=\left\{\left(\bar{d}_{t}, \bar{q}_{t}^{M}, \bar{q}_{t}^{N}\right)\right\}_{t=0}^{\infty}$ generated by $\overline{\boldsymbol{\sigma}}$ where $\bar{q}_{t}^{M}$ and $\bar{q}_{t}^{N}$ are the outputs produced by each firm in period $t$, given that in this period the meeting has and has not been held, respectively. The outcome path $\underline{\mathcal{Z}}$ generated by $\underline{\boldsymbol{\sigma}}$ can be defined in a similar way.

Denote by $\pi^{e}(z, q)$ the expected profit of firm $i$, by $p_{q}^{e}(z)$ the expected market price and by $\mathcal{P}_{q}(z)$ the set of prices that can occur with positive probability in the case where firm $i$ produces the quantity $z$ while each other firm $j$ produces the quantity $q$, i.e.,

$$
\begin{aligned}
\pi^{e}(z, q) & =\pi_{i}^{e}(z, q, . ., q) \\
p_{q}^{e}(z) & =a-z-(n-1) q \\
\mathcal{P}_{q}(z) & =\left[p_{q}^{e}(z)-\Delta, p_{q}^{e}(z)+\Delta\right] .
\end{aligned}
$$

Let $\Pi^{e}(q) \equiv \pi^{e}(q, q), p_{q}^{e} \equiv p_{q}^{e}(q)$ and $\mathcal{P}_{q} \equiv \mathcal{P}_{q}(q)$. It is straightforward to verify that $\Pi^{e}(q) \gtrless \pi^{e}(z, q)$ for any $z \lessgtr q<q^{n}$. This property will be used repeatedly in the subsequent analysis.

Note that in the present setting communication can enhance collusion in two respects.

- First, it can allow firms to sustain harsher punishments. In which case, even

\footnotetext{
${ }^{15}$ The subscript '-i' denotes the vector of the decision variables chosen by all firms except firm $i$.
} 
if it does occur along the equilibrium path, yet it can make collusion easier to sustain.

- Second, it can allow firms to better detect deviations from the collusive output. In which case, it can facilitate collusion even if in the punishment phase firms abandon it for whatever reasons.

Since the goal of the paper is to examine the second effect, the exact value of the punishment is not important. For this reason, a technical assumption is made.

Assumption 1. $k=\left(q^{n}\right)^{2}$.

It states that the static Nash equilibrium yields zero profits. Given that the minmax payoff is also zero, this implies that the strategy $\underline{\sigma}$ takes a particularly simple form: in each period produce $q^{n}$ and propose no communication whatsoever. As a result, one can restrict attention to those equilibria in which in each period firms produce less than $q^{n}$.

\section{Optimal collusive strategy}

Since at $t=0$ there is nothing to reveal yet, while meeting is costly, the optimal collusive strategy $\bar{\sigma}$ must specify no meeting at $t=0$. As Appendix $A$ shows, it must also specify that in each period firms produce the same output $\bar{q}$ regardless of the meeting, i.e., $\bar{q} \equiv \bar{q}_{t}^{M}=\bar{q}_{t}^{N}$ for all $t$, and meet only when the market price falls within the target range of prices $\left[p_{\bar{q}}^{e}-\Delta, \bar{p}\right]$, which is the same for all periods. Furthermore, in case they detect any deviation from $\bar{q}$ or in case some firm waives the meeting when it must be held, it requires firms to switch to the worst sustainable punishment. Formally, the strategy $\bar{\sigma}$ is of the form:

- In period $t=0$ propose no meeting and produce $\bar{q}$;

- In each period $t \geq 1$ proceed as follows:

- if $p_{t-1} \in\left(\bar{p}, p_{\bar{q}}^{e}+\Delta\right]$, then propose no meeting and produce $\bar{q}$;

- if $p_{t-1} \in\left[p_{\bar{q}}^{e}-\Delta, \bar{p}\right]$, then propose to meet;

* if the meeting has taken place and it has revealed that each firm produced $\bar{q}$ in period $t-1$, then produce $\bar{q}$;

* otherwise produce $q^{n}$ and play $\underline{\sigma}$ from the next period onwards;

- if $p_{t-1} \notin\left[p_{\bar{q}}^{e}-\Delta, p_{\bar{q}}^{e}+\Delta\right]$, then play $\underline{\sigma}$ from this period onwards. 
The strategy $\bar{\sigma}$ is thus characterized by the collusive output $\bar{q}$ and the trigger price $\bar{p}$. Specifically, $\bar{q}$ determines both the value of the collusive profit $\Pi^{e}(\bar{q})$ and the set of prices $\mathcal{P}_{\bar{q}}=\left[p_{\bar{q}}^{e}-\Delta, p_{\bar{q}}^{e}+\Delta\right]$ that can occur with positive probability along the equilibrium path, while $\bar{p}$ determines the set of prices $\left[p_{\bar{q}}^{e}-\Delta, \bar{p}\right]$ that trigger the need for a meeting.

Denote by $\bar{\alpha}$ the probability for firms to meet in each period $t \geq 1$, i.e.,

$$
\bar{\alpha}=\operatorname{Pr}\left\{p \in\left[p_{\bar{q}}^{e}-\Delta, \bar{p}\right]\right\}=\frac{\bar{p}-p_{\bar{q}}^{e}+\Delta}{2 \Delta} .
$$

Since, for a given $\bar{q}$, there is one-to-one relationship between $\bar{p}$ and $\bar{\alpha}$, in what follows the equilibrium will be characterized in terms of $(\bar{\alpha}, \bar{q})$ rather than $(\bar{p}, \bar{q})$.

Consider the outcome path $\mathcal{Z}$ induced by the strategy profile $\bar{\sigma}$ and choose some period $t$ in which firms are supposed to meet. Denote by $\bar{V}_{t}^{M}$ the average discounted value of the stream of the expected payoffs generated by $\mathcal{Z}$ from period $t$ onwards. For period $t^{\prime}$ in which firms are not supposed to meet, define $\bar{V}_{t^{\prime}}^{N}$ in a similar way. Since the strategy $\bar{\sigma}$ is stationary, then $\bar{V}_{t}^{N}=\bar{V}^{N}$ and $\bar{V}_{t}^{M}=\bar{V}^{M}$ for all $t$. In which case, it is straightforward to verify that $\bar{V}^{N}$ and $\bar{V}^{M}$ are related as follows:

$$
\begin{aligned}
\bar{V}^{N} & =(1-\delta) \Pi^{e}(\bar{q})+\delta\left(\bar{\alpha} \bar{V}^{M}+(1-\bar{\alpha}) \bar{V}^{N}\right) \\
\bar{V}^{M} & =(1-\delta)\left(\Pi^{e}(\bar{q})-F^{e}\right)+\delta\left(\bar{\alpha} \bar{V}^{M}+(1-\bar{\alpha}) \bar{V}^{N}\right)
\end{aligned}
$$

where $F^{e} \equiv \rho F$. Solving the above system of equations yields:

$$
\begin{aligned}
\bar{V}^{N} & =\Pi^{e}(\bar{q})-\bar{\alpha} \delta F^{e}, \\
\bar{V}^{M} & =\bar{V}^{N}-(1-\delta) F^{e} .
\end{aligned}
$$

For $\bar{\sigma}$ to be an equilibrium strategy, it must be immune to all possible deviations. Consider first deviations in which a firm defects from the collusive output. Note that, if $\bar{\alpha}=1$, then all such deviations will systematically be detected while, if $\bar{\alpha}<1$, then some of them will likely to pass unnoticed. Given this, it will prove useful to distinguish between open and hidden deviations.

In the open deviation a firm clearly reveals that it has cheated. Since cheating can be revealed either by the price realization or by the meeting, it will certainly be detected if it induces zero probability for the realized price to fall within the set of prices, $\left(\bar{p}, p_{\bar{q}}^{e}+\Delta\right]$ triggering no communication. This implies that in the open deviation a firm must produce the output $z$ such that the new set of admissible price realizations $\mathcal{P}_{\bar{q}}(z)$ does not overlap with the set $\left(\bar{p}, p_{\bar{q}}^{e}+\Delta\right]$. Given that firms switch to the worst 
continuation equilibrium following the realization of any $p \notin\left(\bar{p}, p_{\bar{q}}^{e}+\Delta\right]$, such deviation will be unprofitable only if:

$$
(1-\delta) \pi^{e}(z, \bar{q}) \leq \bar{V}^{N}
$$

Substituting (2) into (4) and rearranging the terms yields:

$$
(1-\delta)\left(\pi^{e}(z, \bar{q})-\Pi^{e}(\bar{q})\right) \leq \delta\left(\Pi^{e}(\bar{q})-\bar{\alpha} F^{e}\right)
$$

where $z$ is such that either $p_{\bar{q}}^{e}(z)+\Delta \leq \bar{p}$ or $p_{\bar{q}}^{e}(z)-p_{\bar{q}}^{e}>2 \Delta$.

In contrast, in the hidden deviation a firm seeks to reduce the likelihood of detection of cheating. Towards that end, it produces the output $z \neq \bar{q}$ such that, with some positive probability, the realized price will fall within the interval $\left(\bar{p}, p_{\bar{q}}^{e}+\Delta\right]$. Since this is possible only if the set $\mathcal{P}_{\bar{q}}(z)$ overlaps with the set $\left(\bar{p}, p_{\bar{q}}^{e}+\Delta\right], z$ must satisfy either $\bar{p}<p_{\bar{q}}^{e}(z)+\Delta<p_{\bar{q}}^{e}+\Delta$ or $p_{\bar{q}}^{e}(z)-p_{\bar{q}}^{e}<2 \Delta$. The former condition implies that $0<z-\bar{q}<p_{\bar{q}}^{e}+\Delta-\bar{p}$ while the latter implies that $0<\bar{q}-z \leq 2 \Delta$. In either case, $z$ must differ only marginally from $\bar{q}$.

Denote by $\bar{\beta}(z)$ the probability for any such deviation to be detected, i.e., $\bar{\beta}(z)=$ $\operatorname{Pr}\left\{p \notin\left(\bar{p}, p_{\bar{q}}^{e}+\Delta\right]\right\}$. It is straightforward to verify that, if $p_{\bar{q}}^{e}(z)-p_{\bar{q}}^{e}<2 \Delta$, then:

$$
\bar{\beta}(z)=\bar{\alpha}+\frac{\max \left\{0, p_{\bar{q}}^{e}(z)-\bar{p}-\Delta\right\}}{2 \Delta},
$$

while, if $\bar{p}<p_{\bar{q}}^{e}(z)+\Delta$, then:

$$
\bar{\beta}(z)=\bar{\alpha}+\frac{p_{\bar{q}}^{e}-p_{\bar{q}}^{e}(z)}{2 \Delta} .
$$

Since all detected deviation are followed by the worst sustainable punishment, the hidden deviation will be unprofitable only if:

$$
(1-\delta) \pi^{e}(z, \bar{q})+\delta(1-\bar{\beta}(z)) \bar{V}^{N} \leq(1-\delta) \Pi^{e}(\bar{q})+\delta\left(\bar{\alpha} \bar{V}^{M}+(1-\bar{\alpha}) \bar{V}^{N}\right)
$$

which, in particular, implies:

$$
(1-\delta)\left(\pi^{e}(z, \bar{q})-\Pi^{e}(\bar{q})\right) \leq \delta\left(\bar{\alpha} \bar{V}^{M}+(\bar{\beta}(z)-\bar{\alpha}) \bar{V}^{N}\right)
$$

Note that, since $\bar{\beta}(z) \geq \bar{\alpha}$ and $\pi^{e}(z, \bar{q})<\Pi^{e}(\bar{q})$ for all $z<\bar{q}\left(<q^{n}\right)$, the above condition is trivially satisfied for all $z<\bar{q}$. Thus, in considering (7) it suffices to restrict attention to $z>\bar{q}$. In which case, substituting (2) and (3) into (7) and rearranging 
the terms yields:

$$
(1-\delta)\left(\pi^{e}(z, \bar{q})-\Pi^{e}(\bar{q})\right)+\delta(1-\bar{\beta}(z)) \bar{V}^{N} \leq \delta\left(\Pi^{e}(\bar{q})-\bar{\alpha} F^{e}\right),
$$

where $z$ is such that $0<z-\bar{q}<p_{\bar{q}}^{e}+\Delta-\bar{p}$ and $\bar{\beta}(z)$ is given by (6).

For given $\bar{\alpha},(5)$ and (8) characterize the set of collusive outputs that can be sustained in equilibrium. Although these conditions are formally defined for different $z$, one can neglect this difference while considering them simultaneously. The intuition suggests (and it is proven in Appendix A) that, of the two conditions, (5) is stronger for large output deviations, while the reverse holds for marginal ones. In other words, (5) and (8) are compatible for $z \geq \bar{q}$. Thus, taken together, they can be written as: ${ }^{16}$

$$
\begin{aligned}
\max _{z \geq \bar{q}} \pi^{e}(z, \bar{q})-\Pi^{e}(\bar{q}) & \leq \delta \frac{\Pi^{e}(\bar{q})-\bar{\alpha} F^{e}}{1-\delta}, \\
\max _{z \geq \bar{q}}\left(\pi^{e}(z, \bar{q})-\Pi^{e}(\bar{q})+\delta \frac{1-\bar{\beta}(z)}{1-\delta} \bar{V}^{N}\right) & \leq \delta \frac{\Pi^{e}(\bar{q})-\bar{\alpha} F^{e}}{1-\delta},
\end{aligned}
$$

where $\bar{\beta}(z)$ is given by $(6)$.

Conditions (OD) and (HD) have simple interpretation: the short-term gain from deviating from the collusive output $\bar{q}$ must not exceed the expected loss from abandoning collusion forever afterwards. In this setting, the gains from deviating are twofold. First, given that in Cournot competition outputs act as strategic substitutes, a deviator may gain from increasing its output level. In this respect, (OD) ensures that $\bar{q}$ is immune to all open deviations while (HD) ensures that it is immune to all hidden ones. In the former case, a firm best responds to $\bar{q}$ while, in the latter, it suboptimally increases its output, so that when market demand is high the price mimics a somewhat lower demand state.

Second, given that any detected deviation causes a breakdown of collusion, a deviator may gain from saving on the cost of communication necessarily borne in the collusive phase. More precisely, it then avoids paying $\bar{\alpha} F^{e}$ in all subsequent periods which adds up to saving the amount $\delta \bar{\alpha} F^{e} /(1-\delta)$. This is possible because of the particular form of the punishment considered here, namely, a reversal to the static Nash equilibrium. Although it yields zero payoff, it involves no communication whatsoever.

Consider now the deviation in which a firm waives the meeting when it must be held. Since it is always revealed before firms set their output levels, it can be more immediately punished. But even though firms respond to it by playing the static Nash equilibrium, still it may be profitable because it allows the deviator to save on the cost

\footnotetext{
${ }^{16}$ If $\bar{\alpha}=1$, all deviations are detected with certainty, in which case, only (OD) must be satisfied.
} 
of communication. Thus, each firm will refrain from waiving the meeting only if:

$$
0 \leq(1-\delta)\left(\Pi^{e}(\bar{q})-F^{e}\right)+\delta\left(\bar{\alpha} \bar{V}^{M}+(1-\bar{\alpha}) \bar{V}^{N}\right)
$$

which yields (using (2) and (3)):

$$
0 \leq \bar{V}^{M}=\Pi^{e}(\bar{q})-(1-\delta+\bar{\alpha} \delta) F^{e}
$$

where $\bar{\alpha}>0 .{ }^{17}$

Note that, by using $(2),(3),(6)$ and $p_{\bar{q}}^{e}-p_{\bar{q}}^{e}(z)=z-\bar{q}$, (HD) can be written as:

$$
\max _{z \geq \bar{q}}\left(\pi^{e}(z, \bar{q})-\Pi^{e}(\bar{q})-\frac{z-\bar{q}}{2 \Delta} \frac{\delta}{1-\delta} \bar{V}^{N}\right) \leq \frac{\delta}{1-\delta} \bar{\alpha} \bar{V}^{M}
$$

Since the left hand side of the above condition is always non-negative, (WM) is satisfied whenever (9) is satisfied. Thus, it can be omitted in the subsequent analysis.

Conditions (OD) and (HD) are necessary for the existence of equilibrium in which firms maintain their outputs at the level of $\bar{q}$, and hold meetings on occasion. Appendix $A$ shows that these conditions are also sufficient. This leads to:

Proposition 1 The strategy $\bar{\sigma}(\bar{\alpha}, \bar{q})$ is the optimal collusive strategy wherein $(\bar{\alpha}, \bar{q})$ solves the following problem:

$$
\begin{gathered}
(\bar{\alpha}, \bar{q})=\arg \max _{\alpha, q} \Pi^{e}(q)-\alpha \delta F^{e} \\
\text { s.t. (OD) and (HD) hold. }
\end{gathered}
$$

Proof. See Appendix $A$.

The result has two sides to it: first, firms hold meetings only when the market price falls somewhat below the trigger price and, second, they never revert to a temporary punishment phase. The first feature is similar to the pattern of collusive behavior obtained in Green and Porter (1984) in the sense that, in both models, firms have to take costly action when the market experiences a price decrease. More precisely, in Green and Porter they revert to a temporary price war while here they engage in costly communication. In either case, though the firms' reactions are different, they aim to deter potential deviations from the collusive output.

To gain the intuition for why firms find it optimal to apply a "tail-type" test in choosing whether to meet, suppose instead that they choose to do so when the price falls within the interval $\left[\underline{p}^{\prime}, \bar{p}^{\prime}\right]$ where $\underline{p}^{\prime}>p_{\bar{q}}^{e}-\Delta$ and $\bar{p}^{\prime}<p_{\bar{q}}^{e}+\Delta$. In which case, the

\footnotetext{
${ }^{17}$ If $\bar{\alpha}=0$, then $(\mathrm{WM})$ is irrelevant.
} 
probability of communication would be given by:

$$
\bar{\alpha}^{\prime}=\frac{\bar{p}^{\prime}-\underline{p}^{\prime}}{2 \Delta} \text {. }
$$

Consider a deviation in which a firm marginally increases its output $z$. Assuming that $z$ is such that $\underline{p}^{\prime}<p_{\bar{q}}^{e}(z)+\Delta<\bar{p}^{\prime}$, the probability of detection of such deviation would be given by:

$$
\bar{\beta}^{\prime}(z)=\frac{p_{\bar{q}}^{e}(z)+\Delta-\underline{p}^{\prime}}{2 \Delta}+\frac{p_{\bar{q}}^{e}-p_{\bar{q}}^{e}(z)}{2 \Delta} .
$$

In the above expression, the first term captures the probability that the deviation will be revealed during the meeting while the second one captures the probability that it will be revealed by the price realization. Note that $\bar{\alpha}^{\prime}$ depends only on the difference $\bar{p}^{\prime}-\underline{p}^{\prime}$ while $\bar{\beta}^{\prime}(z)$ increases, as $\underline{p}^{\prime}$ decreases. Thus, by decreasing $\bar{p}^{\prime}$ and $\underline{p}^{\prime}$ while keeping constant the difference $\bar{p}^{\prime}-p^{\prime}$, firms could only increases the probability of detection of deviations without changing the cost of communication. Appendix $A$ also shows that, in the case of other values of $z$, doing so would change neither $\bar{\alpha}^{\prime}$ nor $\bar{\beta}^{\prime}(z)$. As a result, with the tail-type rule applied to the range of admissible price realizations $\mathcal{P}_{\bar{q}}$, firms can do at least as well as with any other rule determining when they must hold a meeting.

The second feature of the collusive strategy obtained here contrasts with the pattern of collusive behavior obtained in Green and Porter. In their setting, communication is not allowed and deviations can never be detected. Consequently, occasional reversions to a price war are needed in order to deter potential deviations. By contrast, in the present setting firms are given the opportunity to communicate which induces a positive probability for any deviation to be detected. ${ }^{18}$ Furthermore, since all detected deviations are most severely punished, the threat of triggering the punishment phase suffices to remove any temptation to deviate. As a result, along the equilibrium path firms maintain the agreed output levels and the meetings just confirm adherence to this agreement. ${ }^{19}$

\footnotetext{
${ }^{18}$ More precisely, deviations can also be detected by the price realizations which fall outside the target set of prices $\mathcal{P}_{\bar{q}}$. This feature is due to the particular distribution of the demand shocks considered here, and it would disappear if $\Delta$ were large enough. For example, if it were $\Delta \geq p \frac{e}{q}$, then firms would meet after realization of any $p \leq \bar{p}$. Hence, it does not alter the qualitative results of the model.

${ }^{19}$ This may seem at odd with the stylized evidence provided by Genesove and Mullin (2001) who argue that cartel meetings do reveal cheating. However, an important feature of their account of cartel organization is that meetings were used not only for detection of deviations but also for renegotiation of retaliation. Since the latter is not allowed in the model, it will only be costly for a cheating firm to attend the meeting.
} 
To better understand how communication facilitates collusion, it will prove useful to consider the situation where firms are not allowed to communicate.

Benchmark: tacit collusion. Denote by $\widehat{\sigma}$ the best collusive strategy in this case. Note that the strategy $\widehat{\sigma}$ can be obtained by using the previous analysis. Specifically, it can be regarded as the strategy $\bar{\sigma}$ which specifies no meetings along the equilibrium path. As a result, $\widehat{\sigma}$ takes a particularly simple form: "produce $\widehat{q}$ as long as $p \in \mathcal{P}_{\widehat{q}}$, otherwise produce $q^{n}$ forever afterwards".

To establish the equilibrium conditions, suppose that all firms adopt the strategy $\bar{\sigma}(\widehat{\alpha}, \widehat{q})$ wherein $\widehat{\alpha}=0$. Since, by construction, no meeting is ever held, condition (WM) is irrelevant. Next, condition (OD) takes the form that looks like the one obtained in the standard deterministic models, i.e.,

$$
\max _{z} \pi^{e}(z, \widehat{q})-\Pi^{e}(\widehat{q}) \leq \frac{\delta}{1-\delta} \Pi^{e}(\widehat{q}) .
$$

The probability of detection of deviations (which are now revealed only if the realized price falls outside the target set of prices $\mathcal{P}_{\widehat{q}}$ ) is equal to:

$$
\widehat{\beta}(z)=\frac{p_{\widehat{q}}^{e}-p_{\widehat{q}}^{e}(z)}{2 \Delta}=\frac{z-\widehat{q}}{2 \Delta} .
$$

Using the above expression and $\widehat{\alpha}=0,(\mathrm{HD})$ can be written as:

$$
\pi^{e}(z, \widehat{q})-\Pi^{e}(\widehat{q}) \leq \frac{\delta}{1-\delta} \frac{z-\widehat{q}}{2 \Delta} \Pi^{e}(\widehat{q})
$$

where $z$ is such that $0 \leq z-\widehat{q} \leq 2 \Delta$. The above condition implies that all marginal deviations will be deterred only if:

$$
2 \Delta(n+1)\left(q^{n}-\widehat{q}\right) \leq \frac{\delta}{1-\delta} \Pi^{e}(\widehat{q}) .
$$

As before, it can be shown that conditions $\left(\mathrm{OD}^{\mathrm{tc}}\right)$ and $\left(\mathrm{HD}^{\mathrm{tc}}\right)$ (where superscript means tacit collusion) are necessary and sufficient for $\widehat{\sigma}$ to be an equilibrium strategy. This leads to:

Proposition 2 The strategy $\widehat{\sigma}(\widehat{q})$ is the optimal collusive strategy without communication wherein $\widehat{q}$ solves the following problem:

$$
\begin{gathered}
\widehat{q}=\arg \max _{q} \Pi^{e}(q) \\
\text { s.t. }\left(O D^{t c}\right) \text { and }\left(H D^{t c}\right) \text { hold. }
\end{gathered}
$$

The proof follows the same steps as for Proposition 1 and, hence, it is omitted. 
Denote by $\widehat{q}_{o}$ (respectively, by $\widehat{q}_{h}$ ) the lowest quantity for which $\left(\mathrm{OD}^{\mathrm{tc}}\right)$ (respectively, $\left.\left(\mathrm{HD}^{\mathrm{tc}}\right)\right)$ is binding. The following proposition establishes the key features of the strategy $\widehat{\sigma}$.

Proposition 3 Suppose that collusive firms do not communicate and punish all detected deviations by a reversal to the static Nash equilibrium forever. Then:

(i) There exists a threshold $\widehat{\delta}_{\min } \in(0,1)$ such that they can sustain collusion if and only if $\delta \geq \widehat{\delta}_{\min }$;

(ii) There exist $\widehat{\delta}_{1}$ and $\widehat{\delta}_{2}$ such that $\widehat{\delta}_{\min }<\widehat{\delta}_{1} \leq \widehat{\delta}_{2}<1$ and $\widehat{q}$ is given by:

$$
\widehat{q}=\left\{\begin{array}{l}
\widehat{q}_{h}, \text { if } \delta \in\left[\widehat{\delta}_{\min }, \widehat{\delta}_{1}\right) \\
\widehat{q}_{o}, \text { if } \delta \in\left[\widehat{\delta}_{1}, \widehat{\delta}_{2}\right) \\
q^{m}, \text { if } \delta \in\left[\widehat{\delta}_{2}, 1\right)
\end{array}\right.
$$

(iii) $\widehat{\delta}_{1}<\widehat{\delta}_{2}$ if $\Delta<a(n-1) / 16 n$ and $\widehat{\delta}_{1}=\widehat{\delta}_{2}$, otherwise.

Proof. See Appendix $B$.

The proposition, in particular, implies that it is always the hidden deviations that do not allow firms to collude when they are sufficiently impatient. Intuitively, in the open deviation a firm optimally responds to the collusive output. This yields a large one-period gain but induces the retaliation for sure. In contrast, in the hidden deviation a firm only suboptimally responds to the collusive output. This in turn yields a lower one-period gain but induces the likelihood for such a deviation to pass unnoticed. When the discount factor is small enough, the gains are almost the same. ${ }^{20}$ As a result, the hidden deviations are more profitable in this case.

Another noteworthy feature of the proposition is that the open deviations can impede tacit collusion only for moderate values of the discount factor and only when the variance of the demand shocks is sufficiently small. This is again quite intuitive: a less uncertain environment increases the probability of detection of the hidden deviations and thereby makes them less profitable in compared to the open ones.

Communication as facilitating device. One can now characterize the impact of communication on collusion. To begin, suppose that the scope for tacit collusion is limited by the open deviations (i.e., $\widehat{q}=\widehat{q}_{o}$ ). In which case, even if firms could communicate, they would optimally refrain from doing so. ${ }^{21}$ This is because the most

\footnotetext{
${ }^{20}$ When the scope for collusion is too limited, even the optimal response involves only a slight modification of the collusive output.

${ }^{21}$ Note that $(\mathrm{OD})$ is stronger than $\left(\mathrm{OD}^{\mathrm{tc}}\right)$ for any $\bar{\alpha}>0$, while both conditions are equivalent for $\bar{\alpha}=0$. Hence, $\Pi^{e}(\bar{q}) \leq \Pi^{e}\left(\widehat{q}_{o}\right)$ for any $(\bar{\alpha}, \bar{q})$ satisfying (OD) which a fortiori implies that $\Pi^{e}(\bar{q})-\bar{\alpha} \delta F^{e} \leq \Pi^{e}\left(\widehat{q}_{o}\right)$.
} 
profitable deviations are those which are already detected with probability one. Accordingly, as long as such deviations are deterred, each firm correctly anticipates that its fellow members will comply with the collusive agreement. This makes communication useless. Consequently, it does not pay for firms to bear the cost of the meetings.

The analysis thus implies that it is only when the scope for tacit collusion is limited by the hidden deviations that firms can benefit from communication. Specifically, we have:

Proposition 4 Suppose that the solution to (P2) is such that $\left(H D^{t c}\right)$ is binding. Then: (i) There exists a threshold of the legal penalty $\bar{F}$ such that the strategy $\bar{\sigma}$ yields a larger expected payoff than the strategy $\widehat{\sigma}$ does whenever $F^{e} \leq \bar{F}$, and it yields a lower expected payoff otherwise;

(ii) $\bar{F} \geq \Pi^{m}$ and $\bar{F}$ is an increasing function of $\Delta$, provided that $\Delta$ is large enough and $n \leq 12$.

\section{Proof. See Appendix $C$.}

The proposition thus implies that when the hidden deviations constrain firms from making collusive profits, communication enables them to further enhance these profits, even though it involves the risk of exposure to legal sanctions. Note that the proposition does not require the legal penalty to be small, rather it suffices that it does not exceed a certain level which tends to increase as demand volatility rises. This is quite intuitive: a more noisy environment renders deviations more profitable which requires firms to meet more often in order to stabilize the cartel. Consequently, a larger penalty is needed to prevent them from doing so. In particular, as the proposition shows, the penalty can even exceed the level of the monopoly profit when demand uncertainty is large enough. ${ }^{22}$

Since the meetings aim to monitor adherence to the collusive strategy, they reduce the scope for deviations and thereby allow firms to further restrict their outputs (i.e., to sustain $\bar{q}$ lower than $\widehat{q}_{h}$ ). However, in choosing the frequency of meetings, $\bar{\alpha}$, they face a trade-off between additional profits to be gained from an increase in the intensity of monitoring and the increased risk that collusion might then be uncovered by the competition authority. On balance, communication will improve collusive profits only if:

$$
\Pi^{e}(\bar{q})-\bar{\alpha} \delta F^{e}>\Pi^{e}\left(\widehat{q}_{h}\right) .
$$

The above condition is readily satisfied for $F^{e}$ small enough. Indeed, as Proposition 3 implies, $\left(\mathrm{OD}^{\mathrm{tc}}\right)$ must necessarily be slack for $\widehat{q}=\widehat{q}_{h}$. Since the functions $\Pi^{e}(\bar{q})$ and

\footnotetext{
${ }^{22}$ Arguably, this is due to the implicit assumption that firms are not liquidity constrained.
} 
$\pi^{e}(z, \bar{q})$ are continuous with respect to their arguments, there must exist $\bar{q}_{\delta}$ such that $\bar{q}_{\delta}<\widehat{q}_{h}$ and the following condition holds:

$$
\max _{z} \pi^{e}\left(z, \bar{q}_{\delta}\right)-\Pi^{e}\left(\bar{q}_{\delta}\right)<\frac{\delta}{1-\delta} \Pi^{e}\left(\bar{q}_{\delta}\right) .
$$

Denote by $\Omega_{h}^{t c}$ the set of the discount factors for which the solution to (P2) implies that $\left(\mathrm{HD}^{\mathrm{tc}}\right)$ is binding. By Proposition $3, \Omega_{h}^{t c}=\left[\widehat{\delta}_{\min }, \widehat{\delta}_{1}\right)$. For any $\delta \in \Omega_{h}^{t c}$, define $F_{\delta}$ as follows:

$$
F_{\delta} \equiv \Pi^{e}\left(\bar{q}_{\delta}\right)-\max \left\{\Pi^{e}\left(\widehat{q}_{h}\right), \frac{1-\delta}{\delta}\left(\max _{z} \pi^{e}\left(z, \bar{q}_{\delta}\right)-\Pi^{e}\left(\bar{q}_{\delta}\right)\right)\right\} .
$$

One can verify that when $F^{e}<\inf \left\{F_{\delta}: \delta \in \Omega_{h}^{t c}\right\}$ there always exists an equilibrium in which firms meet in every period (i.e., set $\bar{\alpha}=1$ ) and maintain their outputs at the level of $\bar{q}_{\delta}$. By construction, in this equilibrium each firm obtains the expected payoff $\Pi^{e}\left(\bar{q}_{\delta}\right)-\delta F^{e}>\Pi^{e}\left(\widehat{q}_{h}\right)$. Thus, the issue is how much $F^{e}$ needs to be small in order for explicit collusion to be more profitable.

In particular, if $\widehat{q}_{h}$ is such that $\Pi^{e}\left(\widehat{q}_{h}\right)-(1-\delta) F^{e}>0$, then in explicit collusion firms can obtain more than $\Pi^{e}\left(\widehat{q}_{h}\right)$ by reducing their outputs just slightly below $\widehat{q}_{h}$ and inducing a small probability of communication. To show this, let $\bar{q}<\widehat{q}_{h}$ and $\bar{\alpha}$ be given by:

$$
\bar{\alpha}=\frac{\Pi^{e}(\bar{q})-\Pi^{e}\left(\widehat{q}_{h}\right)}{\delta F^{e}} .
$$

It suffices to establish that the outcome $(\bar{\alpha}, \bar{q})$, as defined above, is sustainable. ${ }^{23}$ Note first that, for $\bar{\alpha}$ given by (10), the opportunity cost of waiving the meeting is equal to:

$$
\Pi^{e}(\bar{q})-(1-\delta+\bar{\alpha} \delta) F^{e}=\Pi^{e}\left(\widehat{q}_{h}\right)-(1-\delta) F^{e}>0,
$$

which ensures that (WM) is always satisfied. Next, since $\left(\mathrm{OD}^{\mathrm{tc}}\right)$ is not binding for $\widehat{q}=\widehat{q}_{h}$, (OD) must be satisfied for $\bar{\alpha}$ sufficiently small and $\bar{q}$ slightly lower than $\widehat{q}$. Finally, using (6) and $\bar{V}^{N}=\Pi^{e}(\bar{q})-\bar{\alpha} \delta F^{e}=\Pi^{e}\left(\widehat{q}_{h}\right)$, (HD) can be written as:

$$
\begin{aligned}
& \max _{z \geq \bar{q}}\left(\pi^{e}(z, \bar{q})-\Pi^{e}(\bar{q})-\frac{p_{\bar{q}}^{e}-p_{\bar{q}}^{e}(z)}{2 \Delta} \frac{\delta \Pi^{e}\left(\widehat{q}_{h}\right)}{1-\delta}\right) \\
\leq & \frac{\delta}{1-\delta} \bar{\alpha}\left(\Pi^{e}\left(\widehat{q}_{h}\right)-(1-\delta) F^{e}\right) .
\end{aligned}
$$

\footnotetext{
${ }^{23}$ In which case, by continuity, there always exists a sustainable outcome $(\bar{\alpha}, \bar{q})$ which yields an expected payoff strictly larger than $\Pi^{e}\left(\widehat{q}_{h}\right)$.
} 
Note that, since $\bar{q}>q^{m}$, then $\partial \bar{\alpha} / \partial \bar{q}=\left(\delta F^{e}\right)^{-1} \partial \Pi^{e}(\bar{q}) / \partial \bar{q}<0$. Moreover, since (HD ${ }^{\mathrm{tc}}$ ) is binding for $\widehat{q}=\widehat{q}_{h}$, the following conditions must also hold:

$$
\begin{aligned}
& {\left[\max _{z \geq \bar{q}}\left(\pi^{e}(z, \bar{q})-\Pi^{e}(\bar{q})-\frac{p_{\bar{q}}^{e}-p_{\bar{q}}^{e}(z)}{2 \Delta} \frac{\delta \Pi^{e}\left(\widehat{q}_{h}\right)}{1-\delta}\right)\right]_{\bar{q}=\widehat{q}_{h}}=0,} \\
& {\left[\frac{\partial}{\partial \bar{q}} \max _{z \geq \bar{q}}\left(\pi^{e}(z, \bar{q})-\Pi^{e}(\bar{q})-\frac{p_{\bar{q}}^{e}-p \frac{e}{q}(z)}{2 \Delta} \frac{\delta \Pi^{e}\left(\widehat{q}_{h}\right)}{1-\delta}\right)\right]_{\bar{q}=\widehat{q}_{h}}=0 .}
\end{aligned}
$$

Taken together, the above conditions imply that (11) must be satisfied for some $\bar{q}$ slightly lower than $\widehat{q}_{h}$.

In contrast, if $\widehat{q}_{h}$ is such that $\Pi^{e}\left(\widehat{q}_{h}\right)-(1-\delta) F^{e} \leq 0$, then, in order for explicit collusion to be more profitable, firms must considerably reduce their output levels. The analysis is more involved, since not only (HD) but also (OD) must then be treated carefully. As Appendix $C$ shows, even in this case, with communication firms are able to achieve greater collusive profits, provided that the legal penalty does not exceed the level of $\bar{F}$.

Consider now the other side of Proposition 3, viz. the inability of firms to sustain tacit collusion for low values of the discount factor, i.e., for $\delta<\widehat{\delta}_{\text {min }}$. As was explained above, this is because for those discount factors they are unable to deter the hidden deviations. Note however that $\left(\mathrm{HD}^{\mathrm{tc}}\right)$ is binding for $\delta=\widehat{\delta}_{\text {min }}$. In which case, Proposition 4 implies that firms could instead sustain explicit collusion, provided that $F^{e} \leq \bar{F}$. Furthermore, given that conditions (OD) and (HD) are continuous with respect to $\delta$, this must also hold true for some $\delta<\widehat{\delta}_{\text {min }}$ but close enough to $\widehat{\delta}_{\text {min }}$. The following proposition confirms the intuition.

Proposition 5 Suppose that $F^{e}<\bar{F}$. Then, there exists a threshold $\bar{\delta}_{\text {min }} \in\left(0, \widehat{\delta}_{\text {min }}\right)$ such that explicit collusion is sustainable if and only if $\delta \geq \bar{\delta}_{\min }$.

Proof. See Appendix $D$.

The proposition thus implies that, even though communication can help restore the scope for collusion in markets where collusion would not be sustainable otherwise, yet it cannot make collusion sustainable when firms are too impatient.

To gain the intuition behind the result obtained, it is useful to distinguish between the deterrence effect and the value diminishing effect of communication. In the first case, communication reduces the incentives to deviate by increasing the probability of detection of deviations while, in the second case, it reduces the value of collusion by exposing firms to the risk of legal punishment. It turns out that, even though the first effect relaxes the incentive constraints and thereby enlarges the scope for collusion, 
yet the second effect renders collusion unprofitable when the discount factor is small enough.

\section{$5 \quad$ Frequency of meetings}

Although the importance of communication in collusion was recognized long ago, the literature has somehow neglected a number of related issues. How often do firms communicate? Which factors, if any, can influence the frequency of communication? Does it depend on matters to be discussed? This section aims to provide insights into these questions. However, since a closed form solution to problem (P1) cannot be explicitly derived, it is not possible to determine analytically how it varies with the parameters $\delta, \Delta, F^{e}$ and $n$. For this reason, simulations are performed for particular values of these parameters.

Since $\bar{\alpha}$ is the probability for firms to meet in every single period, it is natural to consider it as a measure of the frequency of the meetings (the inverse of $\bar{\alpha}$ is then the mean time between two consecutive meetings). Figure 1 displays the graphs of $\bar{\alpha}$ as a function of $\delta$ calculated for different values of $n$ while holding $\Delta$ and $F^{e}$ fixed. Figure 2 displays the graphs of the same function calculated for different values of $\Delta$ while holding $n$ and $F^{e}$ fixed. ${ }^{24}$

[Insert Figures 1 and 2]

In the figures, each graph is drawn on the interval $\left[\bar{\delta}_{\min }, \bar{\delta}\right]$ where $\bar{\delta}$ is the minimal discount factor for which collusive firms are able to sustain the fully integrated monopoly outcome without communication. In each case, the parameter $\Delta$ has been chosen such that the sustainability of tacit collusion be constrained by the hidden deviations only, i.e., $\Delta>(n-1) / 16 n$, as implied by Proposition 3 .

From inspection of Figure 1 it follows that, as $n$ increases, the graph of $\bar{\alpha}$ shifts rightward and $\bar{\delta}_{\text {min }}$ becomes larger. This suggests that the conventional view (often justified in terms of a Nash equilibrium in an infinitely repeated game in which communication is per se irrelevant) that collusion is easier to sustain the fewer firms are in the market seems to hold even when they engage in meaningful communication. In addition, the figure also shows that in the interval of $\delta$ 's where all the functions are well defined (e.g., in the interval from 0.70 to 0.81$) \bar{\alpha}$ tends to increase, as $n$ rises. Put it differently, firms tend to communicate more often, as a cartel grows in size. Intuitively, in this case each firm obtains a lower market share and therefore it is more tempted

\footnotetext{
${ }^{24}$ In each case $\bar{\alpha}$ is obtained as a solution to (P1). The details of calculations are available upon request.
} 
to deviate by marginally increasing its output. As a result, in order to discipline its members, a cartel increases the intensity of monitoring.

Another noteworthy feature of Figures 1 and 2 is that the function $\bar{\alpha}$ is nonmonotonic: it increases in the neighborhood of $\bar{\delta}_{\text {min }}$ and decreases otherwise. The increasing part of $\bar{\alpha}$ might be explained as follows. For the chosen values of the parameters $F^{e}$ and $\Delta$ it is the hidden deviations that impede collusion when $\delta$ is close enough to $\bar{\delta}_{\min }$. In this case, firms can sustain collusion only by means of monitoring of their private actions. As a result, they increase the frequency of meetings despite the fact that doing so diminishes collusive profits. ${ }^{25}$ In contrast, when $\delta$ is large enough, they are less tempted to deviate which allows a cartel to reduce the intensity of monitoring. This in turn explains the decreasing part of $\bar{\alpha}$.

Lastly, Figure 2 suggests that $\bar{\alpha}$ is an increasing function of the variance of the demand shock $\Delta$. This again seems quite intuitive. A more noisy environment increases the scope for deviations which makes collusion more difficult to sustain. Consequently, in order to enhance the sustainability collusion, more communication and more monitoring are required.

\section{Concluding remarks}

The paper argues that in a situation where the threat of legal prosecution constrains collusive firms from meeting too often, they choose to do so only if the market price falls somewhat below the target price. By using occasional meetings most efficiently, firms are still able to collude in a better way. More precisely, when collusion is already somewhat effective, the meetings allow firms to achieve greater collusive profits. Furthermore, they allow firms to restore the scope for collusion in markets where collusion would not be sustainable otherwise.

The paper also sheds light on the relationship between the frequency of meetings and changes in market environment. In this regard, it delivers important implications for more efficient cartel detection. Specifically, the results suggest that the competition authorities should take into account that cartel participants tend to meet more often in markets where they value future experiences to a lesser degree, they face greater uncertainty or where cartels are bigger in size.

The model builds on the assumption that the probability distribution of the demand shocks is uniform. As a result, the optimal collusive strategy, in this setting, requires

\footnotetext{
${ }^{25}$ Simulations also show that the increasing part of $\bar{\alpha}$ disappears when $\Delta$ and/or $F^{e}$ is small enough. In this case, both (HD) and (OD) rather than (HD) alone govern the sustainability of collusion. In particular, since (OD) is easier to satisfy when $\alpha$ is smaller and/or $\delta$ is larger, the function $\bar{\alpha}$ is found to be strictly decreasing on $\left[\bar{\delta}_{\min }, \bar{\delta}\right]$.
} 
firms to apply a tail-type test in choosing whether to meet. Related to alternative specifications of uncertainty, my conjecture is that a similar strategy should also be optimal for at least those probability distribution functions that satisfy the condition that low prices are more likely to result from a large total output rather than a small one.

The other simplifying assumption is that the competition authority just blindly fights collusion, i.e., it makes no use of either the actual level or the dynamics of prices. Such an approach rules out any strategic interaction between firms and the competition authority. An explicit account for this in a dynamic context is a challenging task, and it is left for future research. 


\section{Appendix}

\section{A. Proof of Proposition 1}

Consider equilibria in which firms condition their play only on the history of commonly observed variables, i.e., market prices, decisions to meet and each other's output levels disclosed during the meetings. Next, consider a history along the equilibrium path leading to a period in which each firm is required to produce the output $q$. Given that $\varepsilon$ is drawn from a bounded support, firms expect the realized price to fall within the interval $\mathcal{P}_{q}$. If instead it falls outside this interval, they infer with probability one that cheating has occurred. But since the firm that has deviated can condition its continuation strategy on its output $z \neq q$, the other firms need to make conjectures about its unobservable action. Moreover, since there is a possibility that they disagree on or form incorrect beliefs about $z$, in the continuation game they can obtain the payoffs which are even lower than the ones that they would obtain in the worst sequential equilibrium.

To avoid technical difficulties related to specification of beliefs in the event of unexpected price realization, in what follows only those strategies which require firms to revert forever to the static Nash equilibrium after the realization of any $p \notin \mathcal{P}_{q}$ will be considered. This guarantees that the continuation-strategy profile is always an equilibrium and therefore the methodology of Abreu et al. $(1986,1990)$ can be applied.

Denote by $\mathcal{V}^{M}$ (respectively, by $\mathcal{V}^{N}$ ) the set of expected payoffs generated by the equilibrium strategies which necessarily require firms to meet (respectively, not to meet) at period $t=0$. Note that $\mathcal{V}^{N} \neq \varnothing$ because playing the static Nash equilibrium in every period constitutes a Nash equilibrium in the game $G^{\infty}(\delta)$. Note also that $\mathcal{V}^{M} \neq \varnothing$ for $F^{e}=0$ because communication enables firms to replace an imperfectmonitoring setting with a perfect-monitoring one. In which case, an equilibrium always exists. Hence, $\mathcal{V}^{M} \neq \varnothing$ at least for sufficiently small values of $F^{e}$. Finally, since meeting is costly, then $\mathcal{V}^{M} \subset \mathcal{V}^{N}$.

Following Abreu et al. (1986, 1990), any equilibrium strategy can be factored into the stage decision variables $d \in \mathcal{D}$ and $q \in \mathcal{Q}$, and the corresponding continuation payoff functions $v(d)$ and $V(p)$. The function $v(d) \in \mathcal{V}^{N}$ ensures that a firm will never gain from waiving the meeting while the function $V(p) \in \mathcal{V}^{N}$ ensures that it will never gain from deviating from the collusive output.

Consider a period on the equilibrium path in which firms are required to produce the output $q$ each. Note first that $V(p)=0$ for all $p \notin \mathcal{P}_{q}$ because the static Nash 
equilibrium yields zero payoff. Next, denote by $\mathcal{P}^{M} \subseteq \mathcal{P}_{q}$ the set of prices that trigger the need for a meeting. Since, by assumption, the meeting will reveal the exact output produced by each firm, the firm's continuation payoff must also depend on whether it has defected from $q$ or not. Accordingly, denote by $V_{\text {dev }}^{M}(p) \in \mathcal{V}^{N}$ its continuation payoff in case it has done so, and by $V^{M}(p) \in \mathcal{V}^{M}$ its continuation payoff in case it has not. Likewise, denote by $\mathcal{P}^{N} \equiv \mathcal{P}_{q} \backslash \mathcal{P}^{M}$ the set of prices triggering no communication, and by $V^{N}(p) \in \mathcal{V}^{N}$ the firm's continuation payoff in this case. It is implied that $V^{M}(p)$ and $V_{\text {dev }}^{M}(p)$ are defined for all $p \in \mathcal{P}^{M}$ while $V^{N}(p)$ is defined for all $p \in \mathcal{P}^{N}$.

Consider now a deviation in which a firm produces an output $z \neq q$. Since, by doing so, it induces the set of feasible price realizations $\mathcal{P}_{q}(z) \neq \mathcal{P}_{q}$, in what follows it will prove useful to define the set $\mathcal{O}_{q}(z) \equiv \mathcal{P}_{q}(z) \cap \mathcal{P}_{q}$. By construction, $\mathcal{O}_{q}(z)$ is the set of prices that can occur with positive probability regardless of whether the firm has deviated or not. As for detection of deviations, three cases need to be distinguished.

In the first case, $p \in \mathcal{P}_{q}(z) \backslash \mathcal{O}_{q}(z)$. Since $\mathcal{P}_{q} \cap \mathcal{P}_{q}(z) \backslash \mathcal{O}_{q}(z)=\varnothing$, this implies that $p \notin \mathcal{P}_{q}$. In other words, the deviation will be revealed by the price realization. In the second case, $p \in \mathcal{P}_{q}(z) \cap \mathcal{P}^{M}$ which implies that the deviation will be revealed during the meeting if the firm chooses to attend it; otherwise, it will be revealed by its refusal to do so. In the third case, $p \in \mathcal{P}_{q}(z) \cap \mathcal{P}^{N}$ which implies that the deviation will pass undetected.

From the above discussion it follows that, if $q$ is produced in some period on the equilibrium path, it must satisfy:

$$
\begin{aligned}
& (1-\delta) \Pi^{e}(q)+\delta\left(\mathrm{E}\left[V^{N}(p) \mid p \in \mathcal{P}^{N}\right]+\mathrm{E}\left[V^{M}(p) \mid p \in \mathcal{P}^{M}\right]\right) \\
\geq & (1-\delta) \pi^{e}(z, q) \\
& +\delta\left(\mathrm{E}\left[V^{N}(p) \mid p \in \mathcal{P}_{q}(z) \cap \mathcal{P}^{N}\right]+\mathrm{E}\left[V_{\text {dev }}^{M}(p) \mid p \in \mathcal{P}_{q}(z) \cap \mathcal{P}^{M}\right]\right),
\end{aligned}
$$

for all $z \in \mathcal{Q}$.

Consider now a period on the equilibrium path in which firms are first required to meet and then produce the output $q$ each. Given that a firm's refusal to meet is commonly known, the other firms can respond immediately to such deviation by modifying their output levels. Denote by $v \in \mathcal{V}^{N}$ the continuation payoff of a firm that refuses to attend the meeting when it must be held. Thus, it will never gain from doing so only if:

$$
(1-\delta)\left(\Pi^{e}(q)-F^{e}\right)+\delta\left(\mathrm{E}\left[V^{M}(p) \mid p \in \mathcal{P}^{M}\right]+\mathrm{E}\left[V^{N}(p) \mid p \in \mathcal{P}^{N}\right]\right) \geq v
$$

Note that, without loss of generality, the set $\mathcal{P}^{M}$ can be represented by collection 
of $s \geq 1$ nonoverlapping intervals, i.e., $\mathcal{P}^{M}=\cup_{i=1}^{s} \mathcal{P}_{i}^{M}$ where $\mathcal{P}_{i}^{M}=\left[p_{i}, p^{i}\right]$ for each $i$ and:

$$
p_{q}-\Delta<p_{1}<p^{1}<\ldots<p_{s}<p^{s}<p_{q}+\Delta \text {. }
$$

Let $p_{0}=p^{0} \equiv p_{q}^{e}-\Delta, p_{s+1}=p^{s+1} \equiv p_{q}^{e}+\Delta$ and $\phi(p) \equiv p / 2 \Delta$. For each $k=0, \ldots, s+1$ define the functions:

$$
\begin{aligned}
\Phi_{k}\left(\mathcal{P}^{M}\right) & \equiv \sum_{i=k}^{s+1} \phi\left(p^{i}-p_{i}\right), \\
\Phi^{k}\left(\mathcal{P}^{M}\right) & \equiv \sum_{i=0}^{k} \phi\left(p^{i}-p_{i}\right) .
\end{aligned}
$$

Also, define the indicator function:

$$
\mathbf{I}_{\{x \in[a, b]\}}=\left\{\begin{array}{l}
1, \text { if } x \in[a, b] \\
0, \text { otherwise. }
\end{array}\right.
$$

Denote by $\alpha\left(\mathcal{P}^{M}\right) \equiv \mathrm{E}\left[p \in \mathcal{P}^{M}\right]$ the probability for firms to meet in the following period. Using the expressions for $\Phi_{k}$ and $\Phi^{k}$, one can show:

$$
\alpha\left(\mathcal{P}^{M}\right)=\Phi_{0}\left(\mathcal{P}^{M}\right)=\Phi^{s+1}\left(\mathcal{P}^{M}\right)
$$

Denote by $\beta\left(z \mid \mathcal{P}^{M}\right)$ the probability of detection of the deviation in which a firm produces an output $z \neq q$, i.e.,

$$
\beta\left(z \mid \mathcal{P}^{M}\right) \equiv \mathrm{E}\left[p \in \mathcal{P}_{q}(z) \backslash \mathcal{O}_{q}(z) \text { or } p \in \mathcal{P}_{q}(z) \cap \mathcal{P}^{M}\right]=\mathrm{E}\left[p \notin \mathcal{P}_{q}(z) \cap \mathcal{P}^{N}\right]
$$

Depending on the value of $z$, two cases need to be distinguished. In the first case, $z$ is such that $\left|p_{q}^{e}-p_{q}^{e}(z)\right|>2 \Delta$. This condition implies that $\mathcal{O}_{q}(z)=\varnothing$ and, therefore, $\beta\left(z \mid \mathcal{P}^{M}\right)=1$, i.e., the deviation will be detected for sure. In the second case, $z$ is such that $\left|p_{q}^{e}-p_{q}^{e}(z)\right| \leq 2 \Delta$. This condition in turn implies that $\mathcal{O}_{q}(z) \neq \varnothing$ and, therefore, the deviation will be detected only with some positive probability. In particular, one can verify that, if $0<p_{q}^{e}(z)-p_{q}^{e} \leq 2 \Delta$, then:

$$
\begin{aligned}
\beta\left(z \mid \mathcal{P}^{M}\right)= & \phi\left(p_{q}^{e}(z)-p_{q}^{e}\right)+\sum_{k=1}^{s+1} \Phi_{k}\left(\mathcal{P}^{M}\right) \mathbf{I}_{\left\{p_{q}^{e}(z)-\Delta \in\left(p^{k-1}, p_{k}\right)\right\}} \\
& +\sum_{k=1}^{s}\left(\phi\left(p^{k}-p_{q}^{e}(z)+\Delta\right)+\Phi_{k+1}\left(\mathcal{P}^{M}\right)\right) \mathbf{I}_{\left\{p_{q}^{e}(z)-\Delta \in\left[p_{k}, p^{k}\right]\right\}},
\end{aligned}
$$


while, if $0<p_{q}^{e}-p_{q}^{e}(z) \leq 2 \Delta$, then:

$$
\begin{aligned}
\beta\left(z \mid \mathcal{P}^{M}\right)= & \phi\left(p_{q}^{e}-p_{q}^{e}(z)\right)+\sum_{k=1}^{s+1} \Phi^{k-1}\left(\mathcal{P}^{M}\right) \mathbf{I}_{\left\{p_{q}^{e}(z)+\Delta \in\left(p^{k-1}, p_{k}\right)\right\}} \\
& +\sum_{k=1}^{s}\left(\phi\left(p_{q}^{e}(z)+\Delta-p_{k}\right)+\Phi^{k-1}\left(\mathcal{P}^{M}\right)\right) \mathbf{I}_{\left\{p_{q}^{e}(z)+\Delta \in\left[p_{k}, p^{k}\right]\right\}} .
\end{aligned}
$$

Lemma A1. $\beta\left(z \mid \mathcal{P}^{M}\right)>\alpha\left(\mathcal{P}^{M}\right)$ for any $z \neq q$.

Proof. Note first that (A3) implies that $\alpha\left(\mathcal{P}^{M}\right)<1$ and:

$$
\begin{aligned}
& \phi\left(p^{k}-p_{q}^{e}+\Delta\right)>\Phi^{k}\left(\mathcal{P}^{M}\right), \\
& \phi\left(p_{q}^{e}+\Delta-p_{k}\right)>\Phi_{k}\left(\mathcal{P}^{M}\right),
\end{aligned}
$$

for any $k \geq 1$. The conclusion thus follows immediately if $\left|p_{q}^{e}-p_{q}^{e}(z)\right|>2 \Delta$, since in this case $\beta\left(z \mid \mathcal{P}^{M}\right)=1$.

Consider the case $0<p_{q}^{e}(z)-p_{q}^{e} \leq 2 \Delta$. Supposing that $p_{q}^{e}(z)-\Delta \in\left(p^{k-1}, p_{k}\right)$ for some $k \geq 1$ and using (A4) and (A6), we have:

$$
\begin{aligned}
\beta\left(z \mid \mathcal{P}^{M}\right) & =\phi\left(p_{q}^{e}(z)-p_{q}^{e}\right)+\Phi_{k}\left(\mathcal{P}^{M}\right)>\phi\left(p^{k-1}-p_{q}^{e}+\Delta\right)+\Phi_{k}\left(\mathcal{P}^{M}\right) \\
& >\Phi^{k-1}\left(\mathcal{P}^{M}\right)+\Phi_{k}\left(\mathcal{P}^{M}\right)=\alpha\left(\mathcal{P}^{M}\right) .
\end{aligned}
$$

Supposing instead that $p_{q}^{e}(z)-\Delta \in\left[p_{k}, p^{k}\right]$ for some $k \geq 1$ and proceeding in a similar way, we have:

$$
\begin{aligned}
\beta\left(z \mid \mathcal{P}^{M}\right) & =\phi\left(p_{q}^{e}(z)-p_{q}^{e}\right)+\phi\left(p^{k}-p_{q}^{e}(z)+\Delta\right)+\Phi_{k+1}\left(\mathcal{P}^{M}\right) \\
& =\phi\left(p^{k}-p_{q}^{e}+\Delta\right)+\Phi_{k+1}\left(\mathcal{P}^{M}\right)>\Phi^{k}\left(\mathcal{P}^{M}\right)+\Phi_{k+1}\left(\mathcal{P}^{M}\right)=\alpha\left(\mathcal{P}^{M}\right)
\end{aligned}
$$

Finally, consider the case $0<p_{q}^{e}-p_{q}^{e}(z) \leq 2 \Delta$. Supposing that $p_{q}^{e}(z)+\Delta \in$ $\left(p^{k-1}, p_{k}\right)$ for some $k \geq 1$ and using (A5) and (A7), we have:

$$
\begin{aligned}
\beta\left(z \mid \mathcal{P}^{M}\right) & =\phi\left(p_{q}^{e}-p_{q}^{e}(z)\right)+\Phi^{k-1}\left(\mathcal{P}^{M}\right)>\phi\left(p_{q}^{e}+\Delta-p_{k}\right)+\Phi^{k-1}\left(\mathcal{P}^{M}\right) \\
& >\Phi_{k}\left(\mathcal{P}^{M}\right)+\Phi^{k-1}\left(\mathcal{P}^{M}\right)=\alpha\left(\mathcal{P}^{M}\right) .
\end{aligned}
$$

Supposing instead that $p_{q}^{e}(z)+\Delta \in\left[p_{k}, p^{k}\right]$ for some $k \geq 1$ and proceeding in a similar way, we have:

$$
\begin{aligned}
\beta\left(z \mid \mathcal{P}^{M}\right) & =\phi\left(p_{q}^{e}-p_{q}^{e}(z)\right)+\phi\left(p_{q}^{e}(z)+\Delta-p_{k}\right)+\Phi^{k-1}\left(\mathcal{P}^{M}\right) \\
& =\phi\left(p_{q}^{e}+\Delta-p_{k}\right)+\Phi^{k-1}\left(\mathcal{P}^{M}\right)>\Phi_{k}\left(\mathcal{P}^{M}\right)+\Phi^{k-1}\left(\mathcal{P}^{M}\right)=\alpha\left(\mathcal{P}^{M}\right) .
\end{aligned}
$$


This completes the proof.

Denote by $\bar{V}^{M}$ the greatest element of $\mathcal{V}^{M}$ and by $\overline{\boldsymbol{\sigma}}^{M}=\left(\bar{\sigma}^{M}, \ldots, \bar{\sigma}^{M}\right)$ the strategy profile generating $\bar{V}^{M}$. Likewise, denote by $\bar{V}^{N}$ the greatest element of $\mathcal{V}^{N}$ and by $\overline{\boldsymbol{\sigma}}^{N}=\left(\bar{\sigma}^{N}, \ldots, \bar{\sigma}^{N}\right)$ the strategy profile generating $\bar{V}^{N}$. Note also that the lowest element of $\mathcal{V}^{N}$ is generated by the strategy profile $\underline{\sigma}=(\underline{\sigma}, \ldots, \underline{\sigma})$ which involves playing the static Nash equilibrium in every period.

Lemma A2. If in some period on the equilibrium path firms meet and produce the output $q$ each, then $q$ must satisfy the following two conditions:

$$
0 \leq(1-\delta)\left(\Pi^{e}(q)-F^{e}\right)+\delta\left(\alpha\left(\mathcal{P}^{M}\right) \bar{V}^{M}+\left(1-\alpha\left(\mathcal{P}^{M}\right)\right) \bar{V}^{N}\right),
$$

and

$$
(1-\delta)\left(\pi^{e}(z, q)-\Pi^{e}(q)\right) \leq \delta\left(\alpha\left(\mathcal{P}^{M}\right) \bar{V}^{M}+\left(\beta\left(z \mid \mathcal{P}^{M}\right)-\alpha\left(\mathcal{P}^{M}\right)\right) \bar{V}^{N}\right)
$$

for all $z \in \mathcal{Q}$.

Proof. By supposition, $q$ satisfies (A1) and (A2). First, consider (A2). Using $V^{M}(p) \leq \bar{V}^{M}, V^{N}(p) \leq \bar{V}^{N}$ and $\alpha\left(\mathcal{P}^{M}\right)=\mathrm{E}\left[p \in \mathcal{P}^{M}\right]$, we have:

$\mathrm{E}\left[V^{M}(p) \mid p \in \mathcal{P}^{M}\right]+\mathrm{E}\left[V^{N}(p) \mid p \in \mathcal{P}^{N}\right] \leq \alpha\left(\mathcal{P}^{M}\right) \bar{V}^{M}+\left(1-\alpha\left(\mathcal{P}^{M}\right)\right) \bar{V}^{N}$.

Taken together, (A10) and $v \geq 0$ imply that (A8) is satisfied whenever (A2) is satisfied.

Consider now (A1). Note that it can be written as:

$$
(1-\delta)\left(\pi^{e}(z, q)-\Pi^{e}(q)\right) \leq \delta\left(D^{M}+D^{N}\right)
$$

where

$$
\begin{aligned}
D^{M} & \equiv \mathrm{E}\left[V^{M}(p) \mid p \in \mathcal{P}^{M}\right]-\mathrm{E}\left[V_{\text {dev }}^{M}(p) \mid p \in \mathcal{P}_{q}(z) \cap \mathcal{P}^{M}\right] \\
D^{N} & \equiv \mathrm{E}\left[V^{N}(p) \mid p \in \mathcal{P}^{N}\right]-\mathrm{E}\left[V^{N}(p) \mid p \in \mathcal{P}_{q}(z) \cap \mathcal{P}^{N}\right] \\
& =\mathrm{E}\left[V^{N}(p) \mid p \in \mathcal{P}^{N} \backslash \mathcal{P}_{q}(z)\right] .
\end{aligned}
$$

Depending on the value of $z$, it will prove useful to distinguish three cases.

Case 1. $z$ is such that $\left|p_{q}^{e}-p_{q}^{e}(z)\right|>2 \Delta$. This condition implies that $\mathcal{O}_{q}(z)=$ $\varnothing$ and therefore $\mathcal{P}_{q}(z) \cap \mathcal{P}^{M}=\mathcal{P}_{q}(z) \cap \mathcal{P}^{N}=\varnothing$. Substituting the latter conditions 
into the right hand side of (A11) and using (A10) yields:

$$
\begin{aligned}
(1-\delta)\left(\pi^{e}(z, q)-\Pi^{e}(q)\right) & \leq \delta\left(\mathrm{E}\left[V^{M}(p) \mid p \in \mathcal{P}^{M}\right]+\mathrm{E}\left[V^{N}(p) \mid p \in \mathcal{P}^{N}\right]\right) \\
& \leq \delta\left(\alpha\left(\mathcal{P}^{M}\right) \bar{V}^{M}+\left(1-\alpha\left(\mathcal{P}^{M}\right)\right) \bar{V}^{N}\right)
\end{aligned}
$$

Since in this case $\beta\left(z \mid \mathcal{P}^{M}\right)=1$, the above condition implies that (A9) is satisfied whenever (A1) is satisfied.

Case 2. $z$ is such that $0 \leq p_{q}^{e}(z)-p_{q}^{e} \leq 2 \Delta$. This condition, in particular, implies that $z \leq q$. Since $D^{M} \geq 0$ and $D^{N} \geq 0$ while $\pi^{e}(z, q) \leq \Pi^{e}(q)$ for all $z \leq q\left(<q^{n}\right)$, then (A11) is trivially satisfied. Note also that the same holds true for (A9) since, by construction, $\beta\left(z \mid \mathcal{P}^{M}\right)=\alpha\left(\mathcal{P}^{M}\right)$ for $z=q$ while, by lemma A1, $\beta\left(z \mid \mathcal{P}^{M}\right)>\alpha\left(\mathcal{P}^{M}\right)$ for $z \neq q$.

Case 3. $z$ is such that $0<p_{q}^{e}-p_{q}^{e}(z) \leq 2 \Delta$. Note first that $V_{d e v}^{M}(p) \geq 0$ and $V^{M}(p) \leq \bar{V}^{M}$ imply:

$$
D^{M} \leq \alpha\left(\mathcal{P}^{M}\right) \bar{V}^{M} .
$$

Next, suppose that $p_{q}^{e}(z)+\Delta \in\left(p^{k-1}, p_{k}\right)$ for some $k$. In which case, using $V^{N}(p) \leq$ $\bar{V}^{N}$, we have:

$$
\begin{aligned}
D^{N} & =\sum_{i=k}^{s} \int_{p^{i}}^{p_{i+1}} V^{N}(p) \frac{d p}{2 \Delta}+\int_{p_{q}^{e}(z)+\Delta}^{p_{k}} V^{N}(p) \frac{d p}{2 \Delta} \\
& \leq\left(\sum_{i=k}^{s} \frac{p_{i+1}-p^{i}}{2 \Delta}+\frac{p_{k}-p_{q}^{e}(z)-\Delta}{2 \Delta}\right) \bar{V}^{N} .
\end{aligned}
$$

On the other hand, using $\alpha\left(\mathcal{P}^{M}\right)=\Phi_{k}\left(\mathcal{P}^{M}\right)+\Phi^{k-1}\left(\mathcal{P}^{M}\right), p^{s+1}=p_{q}^{e}+\Delta$ and (A5), one can show:

$$
\begin{aligned}
\beta\left(z \mid \mathcal{P}^{M}\right)-\alpha\left(\mathcal{P}^{M}\right) & =\phi\left(p^{s+1}-p_{q}^{e}(z)-\Delta\right)-\Phi_{k}\left(\mathcal{P}^{M}\right) \\
& =\sum_{i=k}^{s} \frac{p_{i+1}-p^{i}}{2 \Delta}+\frac{p_{k}-p_{q}^{e}(z)-\Delta}{2 \Delta} .
\end{aligned}
$$

Substituting the above condition into (A13) yields:

$$
D^{N} \leq\left(\beta\left(z \mid \mathcal{P}^{M}\right)-\alpha\left(\mathcal{P}^{M}\right)\right) \bar{V}^{N}
$$

Finally, suppose that $p_{q}^{e}(z)+\Delta \in\left[p_{k}, p^{k}\right]$ for some $k$. By proceeding in a similar 
way, we have:

$$
D^{N}=\sum_{i=k}^{s} \int_{p^{i}}^{p_{i+1}} V^{N}(p) \frac{d p}{2 \Delta} \leq\left(\sum_{i=k}^{s} \frac{p_{i+1}-p^{i}}{2 \Delta}\right) \bar{V}^{N} .
$$

Likewise, using $\alpha\left(\mathcal{P}^{M}\right)=\Phi_{k}\left(\mathcal{P}^{M}\right)+\Phi^{k-1}\left(\mathcal{P}^{M}\right), p_{s+1}=p_{q}^{e}+\Delta$ and (A5), one can show:

$$
\beta\left(z \mid \mathcal{P}^{M}\right)-\alpha\left(\mathcal{P}^{M}\right)=\phi\left(p_{s+1}-p_{k}\right)-\Phi_{k}\left(\mathcal{P}^{M}\right)=\sum_{i=k}^{s} \frac{p_{i+1}-p^{i}}{2 \Delta} .
$$

As before, substituting the above condition into (A15) yields (A14). To complete the proof, note that (A11), (A12) and (A14), taken together, imply that (A9) is satisfied whenever (A1) is satisfied.

Lemma A3. Suppose that (A8) and (A9) are satisfied for some q. Then, there always exists an equilibrium in which in some period on the equilibrium path firms meet and produce the output $q$ each.

Proof. Consider the following strategy $\sigma^{M}$ :

- In period $t=0$ propose to meet; produce $q$ if the meeting has been held, otherwise produce $q^{n}$ and play $\underline{\sigma}$ from the next period onwards;

- In period $t=1$ play $\bar{\sigma}^{N}$ if $p \in \mathcal{P}^{N}$, play $\bar{\sigma}^{M}$ if $p \in \mathcal{P}^{M}$, and play $\underline{\sigma}$ otherwise.

If a firm adheres to this strategy, it will obtain the expected payoff:

$$
(1-\delta)\left(\Pi^{e}(q)-F^{e}\right)+\delta\left(\alpha\left(\mathcal{P}^{M}\right) \bar{V}^{M}+\left(1-\alpha\left(\mathcal{P}^{M}\right)\right) \bar{V}^{N}\right)
$$

In contrast, if it deviates by waiving the meeting, it will save on $F^{e}$ but obtain zero payoffs in all the subsequent periods. Hence, it will refrain from doing so only if (A8) is satisfied. If instead it deviates by producing an output $z \neq q$, it will obtain the expected payoff:

$$
(1-\delta)\left(\pi^{e}(z, q)-F^{e}\right)+\delta\left(1-\beta\left(z \mid \mathcal{P}^{M}\right)\right) \bar{V}^{N}
$$

Hence, it will refrain from doing so only if (A9) is satisfied.

Corollary A1. (A8) and (A9) are necessary and sufficient conditions for firms to meet and produce the output $q$ each in any period on the equilibrium path.

Define the set $\widetilde{\mathcal{P}}^{M}=\cup_{i=1}^{s} \widetilde{\mathcal{P}}_{i}^{M}$ where $\widetilde{\mathcal{P}}_{i}^{M}=\mathcal{P}_{i}^{M}$ for each $i \neq k$ and $\widetilde{\mathcal{P}}_{k}^{M}=\left[\widetilde{p}_{k}, \widetilde{p}^{k}\right]$ where $\widetilde{p}_{k}=p_{k}-\epsilon, \widetilde{p}^{k}=p^{k}-\epsilon$ and $0<\epsilon \leq p^{k-1}-p_{k}$. By construction, $\widetilde{\mathcal{P}}^{M}$ differs 
from $\mathcal{P}^{M}$ only in that the interval $\left[\widetilde{p}_{k}, \widetilde{p}^{k}\right]$ is shifted leftward relative to the interval $\left[p_{k}, p^{k}\right]$ while keeping constant the length of this interval.

Since $p$ is uniformly distributed on the interval $\mathcal{P}_{q}$, the value of $\alpha$ depends only on the sum of the lengths of the intervals $\mathcal{P}_{1}^{M}, \ldots, \mathcal{P}_{s}^{M}$. Hence, $\alpha\left(\mathcal{P}^{M}\right)=\alpha\left(\widetilde{\mathcal{P}}^{M}\right)$. In contrast, the value of $\beta$ also depends on the location of these intervals. Specifically, we have:

Lemma A4. $\beta\left(z \mid \widetilde{\mathcal{P}}^{M}\right) \geq \beta\left(z \mid \mathcal{P}^{M}\right)$ for all $z>q$.

Proof. Since $\beta\left(z \mid \widetilde{\mathcal{P}}^{M}\right)$ differs from $\beta\left(z \mid \mathcal{P}^{M}\right)$ only on $\left[\widetilde{p}_{k}, p^{k}\right]$, then $\beta\left(z \mid \widetilde{\mathcal{P}}^{M}\right)=$ $\beta\left(z \mid \mathcal{P}^{M}\right)$ for all $z$ such that $p_{q}^{e}(z)+\Delta \notin\left[\widetilde{p}_{k}, p^{k}\right]$. In contrast, for all $z$ such that $p_{q}^{e}(z)+\Delta \in\left[\widetilde{p}_{k}, p^{k}\right]$ we have (using (A5)):

$$
\beta\left(z \mid \widetilde{\mathcal{P}}^{M}\right)-\beta\left(z \mid \mathcal{P}^{M}\right)= \begin{cases}\phi\left(p_{q}^{e}(z)+\Delta-\widetilde{p}_{k}\right), & \text { if } p_{q}^{e}(z)+\Delta \in\left[\widetilde{p}_{k}, p_{k}\right), \\ \phi\left(p_{k}-\widetilde{p}_{k}\right), & \text { if } p_{q}^{e}(z)+\Delta \in\left[p_{k}, \widetilde{p}^{k}\right], \\ \phi\left(p^{k}-p_{q}^{e}(z)-\Delta\right), & \text { if } p_{q}^{e}(z)+\Delta \in\left(\widetilde{p}^{k}, p^{k}\right],\end{cases}
$$

which implies that $\beta\left(z \mid \widetilde{\mathcal{P}}^{M}\right) \geq \beta\left(z \mid \mathcal{P}^{M}\right)$.

In the proof of Lemma A2 it was shown that a firm might gain only from those output deviations in which it increases its output level. On the other hand, by Lemma A4, firms can only increase the probability of detection of such deviations if they choose to meet when the price falls within the set $\widetilde{\mathcal{P}}^{M}$ rather than when it falls within the set $\mathcal{P}^{M}$. This yields:

Corollary A2. In solving for the optimal collusive strategy, one can restrict attention to the set of prices triggering communication which has the form $\mathcal{P}^{M}=\left[p_{0}, p\right]$ where $p_{0}=p_{q}^{e}-\Delta$ and $p=p_{0}+\sum_{i=1}^{s}\left(p^{i}-p_{i}\right)$.

For $\mathcal{P}^{M}=\left[p_{q}^{e}-\Delta, p\right]$ denote $\alpha(p, q) \equiv \alpha\left(\mathcal{P}^{M}\right)$ and $\beta(z \mid p, q) \equiv \beta\left(z \mid \mathcal{P}^{M}\right)$. Using $\alpha\left(\mathcal{P}^{M}\right)=\mathrm{E}\left[p \in \mathcal{P}^{M}\right]$, we have $\alpha(p, q)=\phi\left(p-p_{q}^{e}+\Delta\right)$ while, using (A5), we have:

$$
\begin{aligned}
\beta(z \mid p, q)= & \phi\left(p_{q}^{e}-p_{q}^{e}(z)\right)+\phi\left(2 \Delta+p_{q}^{e}(z)-p_{q}^{e}\right) \mathbf{I}_{\left\{p_{q}^{e}(z)+\Delta \in\left[p_{q}^{e}-\Delta, p\right]\right\}} \\
& +\phi\left(p+\Delta-p_{q}^{e}\right) \mathbf{I}_{\left\{p_{q}^{e}(z)+\Delta \in\left(p, p_{q}^{e}+\Delta\right)\right\}} .
\end{aligned}
$$

Note that since, for given $\alpha$ and $q, p$ is uniquely defined (i.e., $p=2 \Delta \alpha+p_{q}^{e}-\Delta$ ), then the outcome in every single period on the equilibrium path can be characterized in terms of $\alpha$ and $q$ rather than $p$ and $q$.

Define the function:

$$
\widehat{\beta}(z \mid \alpha, q) \equiv \alpha+\phi\left(p_{q}^{e}-p_{q}^{e}(z)\right)=1-\frac{\widehat{z}(\alpha, q)-z}{2 \Delta},
$$


where $\widehat{z}(\alpha, q) \equiv q+2 \Delta(1-\alpha)$.

Using the expressions for $\beta(z \mid p, q)$ and $\widehat{\beta}(z \mid \alpha, q)$, one can verify:

$$
\left.\beta(z \mid \alpha, q) \equiv \beta(z \mid p, q)\right|_{p=2 \Delta \alpha+p_{q}^{e}-\Delta}=\min \{1, \widehat{\beta}(z \mid \alpha, q)\}
$$

Define the functions:

$$
\begin{aligned}
G_{o}(z \mid q) & \equiv \pi^{e}(z, q)-\Pi^{e}(q) \\
G_{h}(z \mid \alpha, q) & \equiv G_{o}(z \mid q)+\frac{\delta}{1-\delta}(1-\widehat{\beta}(z \mid \alpha, q)) \bar{V}^{N}
\end{aligned}
$$

Using the expressions for $\pi^{e}(z, q)$ and $\widehat{\beta}(z \mid \alpha, q)$, one can verify:

(i) $G_{o}(z \mid q)$ is a concave function of $z$;

(ii) $G_{h}(z \mid \alpha, q)$ is a concave function of $z$;

(iii) $G_{o}(\widehat{z} \mid q)=G_{h}(\widehat{z} \mid \alpha, q)$;

(iv) $G_{o}(z \mid q) \gtrless G_{h}(z \mid \alpha, q)$ for $z \gtrless \widehat{z}$;

(v) if $\partial_{z} G_{h}(\widehat{z} \mid \alpha, q) \geq 0$ then $\partial_{z} G_{o}(\widehat{z} \mid q)>\partial_{z} G_{h}(\widehat{z} \mid \alpha, q) \geq 0$;

(vi) if $\partial_{z} G_{o}(\widehat{z} \mid q) \leq 0$ then $\partial_{z} G_{h}(\widehat{z} \mid \alpha, q)<\partial_{z} G_{o}(\widehat{z} \mid q) \leq 0$.

Also, denote:

$$
\begin{aligned}
g_{o}(q) & \equiv \max _{z \geq \bar{z}} G_{o}(z \mid q), \\
g_{h}(\alpha, q) & \equiv \max _{q \leq z \leq \widehat{z}} G_{h}(z \mid \alpha, q), \\
G_{o}(q) & \equiv \max _{z \geq q} G_{o}(z \mid q), \\
G_{h}(\alpha, q) & \equiv \max _{z \geq q} G_{h}(z \mid \alpha, q) .
\end{aligned}
$$

Lemma A5. Suppose that $\mathcal{P}^{M}=\left[p_{0}, p\right]$ where $p_{0}=p_{q}^{e}-\Delta$ and $p=2 \Delta \alpha+p_{q}^{e}-\Delta$. Then $q$ satisfies (A9) if and only if it satisfies the following two conditions:

$$
\begin{aligned}
G_{o}(q) & \leq \frac{\delta}{1-\delta}\left(\alpha \bar{V}^{M}+(1-\alpha) \bar{V}^{N}\right), \\
G_{h}(\alpha, q) & \leq \frac{\delta}{1-\delta}\left(\alpha \bar{V}^{M}+(1-\alpha) \bar{V}^{N}\right) .
\end{aligned}
$$

Proof. In the proof of Lemma A2 it was shown that in considering (A9) it suffices to restrict attention to $z \geq q$. In which case, using (A16)-(A19), it is straightforward 
to verify that (A9) boils down to the following two conditions:

$$
\begin{aligned}
g_{o}(q) & \leq \frac{\delta}{1-\delta}\left(\alpha \bar{V}^{M}+(1-\alpha) \bar{V}^{N}\right), \\
g_{h}(\alpha, q) & \leq \frac{\delta}{1-\delta}\left(\alpha \bar{V}^{M}+(1-\alpha) \bar{V}^{N}\right) .
\end{aligned}
$$

Since the choice sets in maximization problems (A20)-(A21) are larger than the respective choice sets in maximization problems (A18)-(A19), then $g_{o}(q) \leq G_{o}(q)$ and $g_{h}(\alpha, q) \leq G_{h}(\alpha, q)$. Thus, any $q$ satisfying (A22)-(A23) must necessarily satisfy (A24)-(A25).

To show that the opposite holds true, consider two cases.

Case 1. (A25) is stronger than (A24), i.e.,

$$
g_{o}(q) \leq g_{h}(\alpha, q)
$$

Note that (A26) can be satisfied only if $\partial_{z} G_{h}(\widehat{z} \mid \alpha, q)<0$. To show this, suppose, to the contrary, that $\partial_{z} G_{h}(\widehat{z} \mid \alpha, q) \geq 0$. This condition and condition (ii), taken together, imply that $G_{h}(z \mid \alpha, q)$ is increasing on $(q, \widehat{z})$ and, therefore, $g_{h}(\alpha, q)=G_{h}(\widehat{z} \mid \alpha, q)$. In turn, this condition and conditions (iii) and (iv), taken together, imply that $g_{h}(\alpha, q)<$ $g_{o}(q)$ which violates $(\mathrm{A} 26)$.

Next, the condition $\partial_{z} G_{h}(\widehat{z} \mid \alpha, q)<0$ and condition (i), taken together, imply that $G_{h}(z \mid \alpha, q)$ is decreasing for all $z>\widehat{z}$. Thus, $G_{h}(\alpha, q)=g_{h}(\alpha, q)$ and, therefore, (A25) and (A23) are equivalent.

Suppose that $\partial_{z} G_{o}(\widehat{z} \mid q)>0$. In this case, condition (i) implies that $G_{o}(z \mid q)$ is increasing for all $z<\widehat{z}$. Thus, $G_{o}(q)=g_{o}(q)$ and, therefore, (A24) and (A22) are equivalent.

Finally, suppose that $\partial_{z} G_{o}(\widehat{z} \mid q) \leq 0$. In this case, condition (i) implies that $G_{o}(z \mid q)$ must have a unique global maximal point at $z_{o} \leq \widehat{z}$. But since $G_{o}(z \mid q)<G_{h}(z \mid \alpha, q)$ for all $z<\widehat{z}$ and $\partial_{z} G_{h}(\widehat{z} \mid \alpha, q)<\partial_{z} G_{o}(\widehat{z} \mid q) \leq 0$, it must be $G_{o}(q)<g_{h}(\alpha, q)$. Thus, (A22) must be satisfied whenever (A25) (alternatively, (A23)) is satisfied.

Case 2. (A24) is stronger than (A25), i.e.,

$$
g_{o}(q) \geq g_{h}(\alpha, q)
$$

Note that $(\mathrm{A} 27)$ can be satisfied only if $\partial_{z} G_{o}(\widehat{z} \mid q)>0$. To show this, suppose, to the contrary, that $\partial_{z} G_{o}(\widehat{z} \mid q) \leq 0$. In which case, since $G_{o}(z \mid q)$ is concave, it must be decreasing for all $z>\widehat{z}$ and, therefore, $g_{o}(q)=G_{o}(\widehat{z} \mid q)$. This condition and conditions (iii) and (iv), taken together, imply that $g_{o}(q)<g_{h}(\alpha, q)$ which violates (A27). 
As before, in case $\partial_{z} G_{o}(\widehat{z} \mid q)>0$, we have $G_{o}(q)=g_{o}(q)$ which implies that (A24) and (A22) are equivalent. Likewise, in case $\partial_{z} G_{h}(\widehat{z} \mid \alpha, q)<0$, we have $G_{h}(\alpha, q)=$ $g_{h}(\alpha, q)$ which implies that (A25) and (A23) are equivalent.

Finally, suppose that $\partial_{z} G_{h}(\widehat{z} \mid \alpha, q) \geq 0$. In this case, condition (i) implies that $G_{h}(z \mid \alpha, q)$ must have a unique global maximal point at $z_{h} \geq \widehat{z}$. But since $G_{o}(z \mid q)>$ $G_{h}(z \mid \alpha, q)$ for all $z>\widehat{z}$ and $\partial_{z} G_{o}(\widehat{z} \mid q)>\partial_{z} G_{h}(\widehat{z} \mid \alpha, q) \geq 0$, it must be $g_{o}(q)>$ $G_{h}(\alpha, q)$. Thus, (A23) must be satisfied whenever (A24) (alternatively, (A22)) is satisfied.

Define the function:

$$
V^{N}(\alpha, q) \equiv(1-\delta) \Pi^{e}(q)+\delta\left(\alpha \bar{V}^{M}+(1-\alpha) \bar{V}^{N}\right)
$$

and denote:

$$
\begin{gathered}
\left(\alpha^{*}, q^{*}\right) \equiv \arg \max _{\alpha, q} V^{N}(\alpha, q) \\
\text { s.t. (A22) and (A23) hold. }
\end{gathered}
$$

Lemma A6. The optimal collusive strategy $\bar{\sigma}$ implies that in every period on the equilibrium path firms produce the same output $q^{*}$ and meet only if the price falls within the interval $\left[p_{q^{*}}^{e}-\Delta, p^{*}\right]$ where $p^{*} \leq p_{q^{*}}^{e}+\Delta$.

Proof. Set $p=2 \Delta \alpha+p_{q}^{e}-\Delta$ and consider the following strategy $\sigma^{N}(\alpha, q)$ :

- In period $t=0$ propose no meeting and produce $q$;

- In period $t=1$ play $\bar{\sigma}^{N}$ if $p \in\left(p, p_{q}^{e}+\Delta\right]$, play $\bar{\sigma}^{M}$ if $p \in\left[p_{q}^{e}-\Delta, p\right]$, otherwise, play $\underline{\sigma}$.

By construction, $\sigma^{N}(\alpha, q)$ yields the expected payoff $V^{N}(\alpha, q)$. By Lemma A3, $\sigma^{N}(\alpha, q)$ is an equilibrium strategy whenever $(\alpha, q)$ satisfies (A9). In which case, $V^{N}(\alpha, q) \in \mathcal{V}^{N}$ and, therefore, $V^{N}(\alpha, q) \leq \bar{V}^{N}$ for all $(\alpha, q)$ satisfying (A9). In particular, $V^{N}\left(\alpha^{*}, q^{*}\right) \leq \bar{V}^{N}$ since, by Lemma A5, $\left(\alpha^{*}, q^{*}\right)$ satisfies (A9).

Consider the outcome $\left(\bar{\alpha}^{N}, \bar{q}^{N}\right)$ generated by $\bar{\sigma}^{N}$ in period $t=0$. By Lemma A5, it must satisfy (A22) and (A23). In which case, using the definition of $\left(\alpha^{*}, q^{*}\right)$, we must have $V^{N}\left(\bar{\alpha}^{N}, \bar{q}^{N}\right) \leq V^{N}\left(\alpha^{*}, q^{*}\right)$. Since, by construction, $\sigma^{N}\left(\bar{\alpha}^{N}, \bar{q}^{N}\right)=\bar{\sigma}^{N}$, then $\bar{V}^{N} \leq V^{N}\left(\alpha^{*}, q^{*}\right) \leq \bar{V}^{N}$ which implies:

$$
V^{N}\left(\alpha^{*}, q^{*}\right)=\bar{V}^{N} \text {. }
$$

Next, consider the following strategy $\sigma^{M}(\alpha, q)$ :

- In period $t=0$ propose to meet; produce $q$ if the meeting has taken place, otherwise produce $q^{n}$ and play $\underline{\sigma}$ from the next period onwards; 
- In period $t=1$ proceed as the strategy $\sigma^{N}(\alpha, q)$ requires to do from this period onwards.

By construction, $\sigma^{M}(\alpha, q)$ yields the expected payoff $V^{M}(\alpha, q)=V^{N}(\alpha, q)-(1-$ $\delta) F^{e}$. By Lemma A3, if $(\alpha, q)$ satisfies (A8) and (A9) then $\sigma^{M}(\alpha, q)$ is an equilibrium strategy. In which case, $V^{M}(\alpha, q) \in \mathcal{V}^{M}$ and, therefore, $V^{M}(\alpha, q) \leq \bar{V}^{M}$ for all $(\alpha, q)$ satisfying (A8) and (A9).

Note that the set of outcomes $(\alpha, q)$ satisfying (A8) and (A9) is at least as large as the set of outcomes $(\alpha, q)$ satisfying (A9) only. Lemma A5 and the fact that (A8) is equivalent to $V^{N}(\alpha, q)-(1-\delta) F^{e} \geq 0$ imply that the former set is non empty only if $\left(\alpha^{*}, q^{*}\right)$ satisfies $(\mathrm{A} 8)$, i.e., $V^{N}\left(\alpha^{*}, q^{*}\right)-(1-\delta) F^{e} \geq 0$. In which case, using the definition of $\bar{V}^{M}$, we must have $V^{M}\left(\alpha^{*}, q^{*}\right) \leq \bar{V}^{M}$.

As before, consider the outcome $\left(\bar{\alpha}^{M}, \bar{q}^{M}\right)$ generated by $\bar{\sigma}^{M}$ in period $t=0$. By Lemma A2, $\left(\bar{\alpha}^{M}, \bar{q}^{M}\right)$ must satisfy (A8) and (A9). In which case, using the definition of $\left(\alpha^{*}, q^{*}\right)$, we must have $V^{M}\left(\bar{\alpha}^{M}, \bar{q}^{M}\right) \leq V^{M}\left(\alpha^{*}, q^{*}\right)$. Since, by construction, $\sigma^{M}\left(\bar{\alpha}^{M}, \bar{q}^{M}\right)=\bar{\sigma}^{M}$, then $\bar{V}^{M} \leq V^{M}\left(\alpha^{*}, q^{*}\right) \leq \bar{V}^{M}$ which implies:

$$
V^{M}\left(\alpha^{*}, q^{*}\right)=\bar{V}^{M} \text {. }
$$

Using (A28), (A29) and (A30), it is straightforward to verify that $\bar{V}^{N}=\Pi^{e}\left(q^{*}\right)-$ $\alpha^{*} \delta F^{e}$ which is possible only if in all periods on the equilibrium path firms produce the same output $q^{*}$ and meet only if the price falls within the interval $\left[p_{q^{*}}^{e}-\Delta, p^{*}\right]$ where $\bar{p}=2 \Delta \alpha^{*}+p_{q^{*}}^{e}-\Delta$. To complete the proof, it suffices to note that $\bar{\sigma}=\bar{\sigma}^{N}$ since $\bar{V}^{N}>\bar{V}^{M}$.

Lemma A7. $\bar{V}^{N}=\Pi^{e}(\bar{q})-\bar{\alpha} \delta F^{e}$ where $(\bar{\alpha}, \bar{q})$ solves $(P 1)$.

Proof. Since $\sigma(\bar{\alpha}, \bar{q})$ (which is defined in the text) is an equilibrium strategy then $\Pi^{e}(\bar{q})-\bar{\alpha} \delta F^{e} \in \mathcal{V}^{N}$ and therefore:

$$
\Pi^{e}(\bar{q})-\bar{\alpha} \delta F^{e} \leq \bar{V}^{N}
$$

where $\bar{V}^{N}=\Pi^{e}\left(q^{*}\right)-\alpha^{*} \delta F^{e}$, by Lemma A6.

On the other hand, since the outcome path induced by $\sigma\left(\alpha^{*}, q^{*}\right)$ generates the expected payoffs $\bar{V}^{N}=\Pi^{e}\left(q^{*}\right)-\alpha^{*} \delta F^{e}$ and $\bar{V}^{M}=\bar{V}^{N}-(1-\delta) F^{e}$, then $\left(\alpha^{*}, q^{*}\right)$ must satisfy (OD) and (HD). Given that $(\bar{\alpha}, \bar{q})$ solves (P1), we must then have:

$$
\Pi^{e}(\bar{q})-\bar{\alpha} \delta F^{e} \geq \bar{V}^{N}
$$

Taken together, (A31) and (A32) imply that $\bar{V}^{N}=\Pi^{e}(\bar{q})-\bar{\alpha} \delta F^{e}$. 


\section{B. Proof of Proposition 3}

Using the expressions for $\pi^{e}(z, q)$ and $\Pi^{e}(q)$, one can verify:

$$
\max _{z \geq q} \pi^{e}(z, \widehat{q})-\Pi^{e}(\widehat{q})=\left(\frac{1+n}{2}\right)^{2}\left(q^{n}-\widehat{q}\right)^{2}
$$

In what follows, it will prove useful to write $\Pi^{e}(q)$ as (using Assumption 1):

$$
\Pi^{e}(q)=\left(q^{n}-q\right)\left((n-1) q^{n}-n\left(q^{n}-q\right)\right) .
$$

Taken together, (B1) and (B2) imply that the set of outputs satisfying (OD $\left.{ }^{\mathrm{tc}}\right)$ is the interval $\left[\widehat{q}_{o}, q^{n}\right]$. Likewise, the set of outputs satisfying $\left(\mathrm{HD}^{\mathrm{tc}}\right)$ is the interval $\left[\widehat{q}_{h}, q^{n}\right]$. It is straightforward to verify that $\widehat{q}_{o} \leq q^{n}$ for any $\delta \in(0,1)$. In contrast, $\widehat{q}_{h} \leq q^{n}$ if and only if:

$$
\begin{aligned}
0 & \leq\left[\frac{\partial}{\partial \widehat{q}}\left(2 \Delta(n+1)\left(q^{n}-\widehat{q}\right)-\frac{\delta}{1-\delta} \Pi^{e}(\widehat{q})\right)\right]_{\widehat{q}=q^{n}} \\
& =-2 \Delta(n+1)+\frac{\delta}{1-\delta}(n-1) q^{n},
\end{aligned}
$$

which is equivalent to $\delta \geq \widehat{\delta}_{\text {min }}$ where $\widehat{\delta}_{\text {min }}$ is given by:

$$
\widehat{\delta}_{\min } \equiv \frac{1}{1+\frac{(n-1) q^{n}}{2(n+1) \Delta}}
$$

Hence, the set of outputs satisfying both $\left(\mathrm{OD}^{\mathrm{tc}}\right)$ and $\left(\mathrm{HD}^{\mathrm{tc}}\right)$ is non empty if and only if $\delta \geq \widehat{\delta}_{\min }$. In which case, it is given by the interval $\left[\max \left\{\widehat{q}_{o}, \widehat{q}_{h}\right\}, q^{n}\right]$. Since $\Pi^{e}(q)$ decreases on $\left[q^{m}, q^{n}\right]$, the solution to (P2) takes the form:

$$
\widehat{q}=\max \left\{q^{m}, \widehat{q}_{o}, \widehat{q}_{h}\right\}
$$

Next, the condition $\widehat{q}_{h} \leq \widehat{q}_{o}$ holds only if $\left(\mathrm{OD}^{\mathrm{tc}}\right)$ is stronger than $\left(\mathrm{HD}^{\mathrm{tc}}\right)$ for $\widehat{q}=\widehat{q}_{h}$, i.e.,

$$
\left(\frac{1+n}{2}\right)^{2}\left(q^{n}-\widehat{q}_{h}\right)^{2} \geq 2 \Delta(n+1)\left(q^{n}-\widehat{q}_{h}\right),
$$

which yields $\widehat{q}_{h} \leq q^{n}-8 \Delta /(n+1)$. In turn, this condition holds only for those $\delta$ 's which satisfy:

$$
\left[2 \Delta(n+1)\left(q^{n}-\widehat{q}\right)-\frac{\delta}{1-\delta} \Pi^{e}(\widehat{q})\right]_{\widehat{q}=q^{n}-\frac{8 \Delta}{n+1}} \leq 0
$$


which yields $\delta \geq \widehat{\delta}$ where $\widehat{\delta}$ is given by:

$$
\widehat{\delta} \equiv\left(\left(\frac{n-1}{n+1}\right)^{2}+\frac{(n-1) q^{n}}{2(n+1) \Delta}\right)^{-1} .
$$

Using $\left(\mathrm{OD}^{\mathrm{tc}}\right)$ and $\left(\mathrm{HD}^{\mathrm{tc}}\right)$, it is straightforward to verify that $\widehat{q}_{o}$ and $\widehat{q}_{h}$ are decreasing functions of $\delta$. Therefore, $\widehat{q}_{h} \lessgtr \widehat{q}_{o}$ for $\delta \gtrless \widehat{\delta}$.

Denote by $\widehat{\delta}_{h}^{m}$ (respectively, by $\widehat{\delta}_{o}^{m}$ ) the discount factor for which $\widehat{q}_{h}=q^{m}$ (respectively, $\left.\widehat{q}_{o}=q^{m}\right)$. Routing calculations yield:

$$
\begin{aligned}
& \widehat{\delta}_{h}^{m} \equiv\left(1+\frac{(n-1) q^{n}}{4(n+1) \Delta}\right)^{-1} \\
& \widehat{\delta}_{o}^{m} \equiv\left(1+n\left(\frac{2}{n+1}\right)^{2}\right)^{-1} .
\end{aligned}
$$

It is straightforward to verify that $\widehat{\delta}_{h}^{m}<\widehat{\delta}_{o}^{m}$ if and only if:

$$
\Delta<\frac{(n-1)(n+1)}{16 n} q^{n}=\frac{a(n-1)}{16 n} .
$$

Note also that the condition $\max \left\{q^{m}, \widehat{q}_{o}, \widehat{q}_{h}\right\}=\widehat{q}_{o}$ is possible if and only if:

$$
\widehat{\delta}<\widehat{\delta}_{h}^{m} \Leftrightarrow \Delta<\frac{(n-1)(n+1)}{16 n} q^{n}=\frac{a(n-1)}{16 n} .
$$

To complete the proof, it suffices to set $\widehat{\delta}_{1}=\min \left\{\widehat{\delta}, \widehat{\delta}_{h}^{m}\right\}$ and $\widehat{\delta}_{2}=\max \left\{\widehat{\delta}_{1}, \widehat{\delta}_{o}^{m}\right\}$.

\section{Proof of Proposition 4}

Since $\widehat{q}_{h}(\delta)$ decreases from $q^{n}$ to $q^{m}$ on $\left[\widehat{\delta}_{\min }, \widehat{\delta}_{h}^{m}\right]$ while $\Pi^{e}(q)$ decreases on $\left[q^{m}, q^{n}\right]$, then $\Pi^{e}\left(\widehat{q}_{h}(\delta)\right)$ increases from 0 to $\Pi^{m}$ on $\left[\widehat{\delta}_{\min }, \widehat{\delta}_{h}^{m}\right]$. For a given $F^{e}$, define the set $\widehat{\Omega}_{F}$ as:

$$
\widehat{\Omega}_{F} \equiv\left\{\delta: \delta \in\left[\widehat{\delta}_{\min }, \widehat{\delta}_{h}^{m}\right] \text { and }(1-\delta) F^{e} \geq \Pi^{e}\left(\widehat{q}_{h}(\delta)\right)\right\}
$$

In the case $\delta \notin \widehat{\Omega}_{F}$ the proof is given in the text. Thus, only the case $\delta \in \widehat{\Omega}_{F}$ needs to be considered. In addition, since communication can enhance collusion only if it allows firms to sustain the output $\bar{q}<\widehat{q}_{h}$, in what follows the analysis will be restricted to those values of $\bar{q}$ only.

In this case, the fact that $\left(\mathrm{HD}^{\mathrm{tc}}\right)$ is binding for $\widehat{q}_{h}$, in particular, implies that $\bar{q}$ must satisfy $2 \Delta(n+1)\left(q^{n}-\bar{q}\right)>\delta \Pi^{e}(\bar{q}) /(1-\delta)$. Using this condition and replacing 
$\pi^{e}(z, q)$ and $\bar{V}^{N}$ by their expressions, the left hand side of (9) takes the form:

$$
\begin{aligned}
& \max _{z \geq \bar{q}}\left((n+1)\left(q^{n}-\bar{q}\right)-\frac{\delta}{1-\delta} \frac{\Pi^{e}(\bar{q})-\bar{\alpha} \delta F^{e}}{2 \Delta}-(z-\bar{q})\right)(z-\bar{q}) \\
= & \frac{1}{4}\left((n+1)\left(q^{n}-\bar{q}\right)-\frac{\delta}{1-\delta} \frac{\Pi^{e}(\bar{q})-\bar{\alpha} \delta F^{e}}{2 \Delta}\right)^{2} .
\end{aligned}
$$

Since the above expression is strictly positive, (9) can be satisfied only if its right hand side is also strictly positive, i.e., only if $\bar{\alpha}>0$ and $\Pi^{e}(\bar{q})-\bar{\alpha} \delta F^{e}>(1-\delta) F^{e}$. In which case, using the fact that $(1-\delta) F^{e} \geq \Pi^{e}\left(\widehat{q}_{h}\right)$ for any $\delta \in \widehat{\Omega}_{F}$, one obtains $\Pi^{e}(\bar{q})-\bar{\alpha} \delta F^{e}>\Pi^{e}\left(\widehat{q}_{h}\right)$ for any $(\bar{\alpha}, \bar{q})$ satisfying $(9)$.

Define the function:

$$
g(q, \delta, n) \equiv \Pi^{e}(q)-\frac{1-\delta}{\delta}\left(\max _{z} \pi^{e}(z, q)-\Pi^{e}(q)\right) .
$$

Using (B1) and (B2), $g(q, \delta, n)$ can be written as:

$$
g(q, \delta, n)=\left(q^{n}-q\right)\left((n-1) q^{n}-\left(n+\frac{1-\delta}{\delta} \frac{(1+n)^{2}}{4}\right)\left(q^{n}-q\right)\right) .
$$

For a given vector of model parameters $\kappa \equiv\left(\delta, F^{e}, \Delta, n\right)$, define the functions:

$$
\begin{aligned}
\Phi(\alpha, q, \kappa) \equiv & \alpha-\frac{g(q, \delta, n)}{F^{e}} \\
\Psi(\alpha, q, \kappa) \equiv & \frac{1}{4}\left((n+1)\left(q^{n}-q\right)-\frac{\delta}{1-\delta} \frac{\Pi^{e}(q)-\alpha \delta F^{e}}{2 \Delta}\right)^{2} \\
& -\frac{\alpha \delta\left(\Pi^{e}(q)-(1-\delta+\alpha \delta) F^{e}\right)}{1-\delta}
\end{aligned}
$$

By rearranging the terms, $\Psi(\bar{\alpha}, \bar{q}, \boldsymbol{\kappa})$ can be written as:

$$
\begin{aligned}
\Psi(\alpha, q, \boldsymbol{\kappa})= & \frac{1}{(1-\delta) F^{e}}\left(\left(\delta F^{e}\right)^{2}(1+\tau(\boldsymbol{\kappa})) \alpha^{2}\right. \\
& \left.-\left(\delta F^{e}\right)(d(q, \boldsymbol{\kappa})-2 \tau(\boldsymbol{\kappa}) b(q, \boldsymbol{\kappa})) \alpha+\tau(\boldsymbol{\kappa}) b^{2}(q, \boldsymbol{\kappa})\right)
\end{aligned}
$$

where

$$
\begin{aligned}
b(q, \boldsymbol{\kappa}) & \equiv \frac{2 \Delta(1-\delta)}{\delta}(n+1)\left(q^{n}-q\right)-\Pi^{e}(q), \\
d(q, \boldsymbol{\kappa}) & \equiv \Pi^{e}(q)-(1-\delta) F^{e} \\
\tau(\boldsymbol{\kappa}) & \equiv\left(\frac{\delta}{4 \Delta}\right)^{2} \frac{F^{e}}{1-\delta} .
\end{aligned}
$$


Using (B2) and $(n-1) q^{n}=2 \Delta(n+1)\left(1-\widehat{\delta}_{\min }\right) / \widehat{\delta}_{\min }, b(q, \boldsymbol{\kappa})$ can be written as:

$$
b(q, \boldsymbol{\kappa})=\left(q^{n}-q\right)\left(n\left(q^{n}-q\right)+2 \Delta(n+1) \frac{\widehat{\delta}_{\min }-\delta}{\widehat{\delta}_{\min } \delta}\right) .
$$

Again, using (B2) and also $\Pi^{m}=\left((n-1) q^{n}\right)^{2} / 4 n, d(q, \boldsymbol{\kappa})$ can be written as:

$$
d(q, \boldsymbol{\kappa})=n\left(\left(q^{n}-q\right)-x_{(-)}(\boldsymbol{\kappa})\right)\left(x_{(+)}(\boldsymbol{\kappa})-\left(q^{n}-q\right)\right),
$$

where

$$
x_{( \pm)}(\boldsymbol{\kappa}) \equiv \frac{(n-1) q^{n}}{2 n}\left(1 \pm \sqrt{1-\frac{(1-\delta) F^{e}}{\Pi^{m}}}\right) .
$$

By construction, $(\bar{\alpha}, \bar{q})$ satisfies (OD) and (HD) if and only if it satisfies:

$$
\begin{aligned}
\Phi(\bar{\alpha}, \bar{q}, \kappa) & \leq 0 \\
\Psi(\bar{\alpha}, \bar{q}, \kappa) & \leq 0 .
\end{aligned}
$$

To prove the proposition, it suffices to show that there exists a threshold $\bar{F}$ such that for $F^{e} \leq \bar{F}$ and $\delta \in \widehat{\Omega}_{F}$ there always exists $(\bar{\alpha}, \bar{q})$ satisfying (C9) and (C10). Since (C9) can be satisfied for any $F^{e}$, the necessarily condition for the threshold $\bar{F}$ to exist is that $(\mathrm{C} 10)$ cannot be satisfied for some $F^{e}>0$.

Using the expressions for $\Phi(\cdot)$ and $\Psi(\cdot)$, it is straightforward to verify that $\partial \Psi / \partial \delta<$ $0, \partial \Phi / \partial \delta<0, \partial \Psi / \partial F^{e}>0$ and $\partial \Phi / \partial F^{e}>0$. The first two conditions, in particular, imply that if (C9) and (C10) are satisfied for $\delta=\inf \widehat{\Omega}_{F}=\widehat{\delta}_{\text {min }}$, then a fortiori they will be satisfied for any $\delta>\widehat{\delta}_{\text {min }}$ (provided that the other parameters are held fixed). Thus, for the rest of the analysis it will assumed that $\delta=\widehat{\delta}_{\text {min }}$.

Denote $\widehat{\boldsymbol{\kappa}} \equiv\left(\widehat{\delta}_{\min }, F^{e}, \Delta, n\right)$ and $\widehat{\tau} \equiv \tau(\widehat{\boldsymbol{\kappa}})$. Using (B3) and $\Pi^{m}=\left((n-1) q^{n}\right)^{2} / 4 n$, one can verify:

$$
\widehat{\tau}=\frac{(n+1)^{2}}{16 n \Pi^{m}} \frac{F^{e}}{1+\frac{2(n+1) \Delta}{(n-1) q^{n}}} .
$$

Since $\Psi(\bar{\alpha}, \bar{q}, \widehat{\boldsymbol{\kappa}})$ is a quadratic function of $\alpha$, then, for given $\bar{q}$ and $\widehat{\boldsymbol{\kappa}},(\mathrm{C} 10)$ can be satisfied in the relevant range in which $\bar{\alpha}>0$ only if the equation $\Psi(\bar{\alpha}, \bar{q}, \widehat{\boldsymbol{\kappa}})=0$ has real roots. In turn, this is possible only if:

$$
\begin{aligned}
d(\bar{q}, \widehat{\boldsymbol{\kappa}})-2 \widehat{\tau} b(\bar{q}, \widehat{\boldsymbol{\kappa}}) & >0 \\
(d(\bar{q}, \widehat{\boldsymbol{\kappa}})-2 \widehat{\tau} b(\bar{q}, \widehat{\boldsymbol{\kappa}}))^{2} & \geq 4 \widehat{\tau}(1+\widehat{\tau}) b^{2}(\bar{q}, \widehat{\boldsymbol{\kappa}}),
\end{aligned}
$$


which imply:

$$
d(\bar{q}, \widehat{\boldsymbol{\kappa}}) \geq \xi(\widehat{\tau}) b(\bar{q}, \widehat{\boldsymbol{\kappa}}),
$$

where $\xi(\tau) \equiv 2 \tau(1+\sqrt{1+1 / \tau})$.

Using $(\mathrm{C} 6), b(q, \boldsymbol{\kappa})$ evaluated at $\delta=\widehat{\delta}_{\text {min }}$ yields:

$$
b(\bar{q}, \widehat{\kappa})=n\left(q^{n}-\bar{q}\right)^{2}
$$

Substituting $(\mathrm{C} 7)$ and $(\mathrm{C} 13)$ into $(\mathrm{C} 12)$ and rearranging the terms leads to (after dividing by $n)$ :

$$
(1+\xi(\widehat{\tau})) \bar{x}^{2}-\left(\widehat{x}_{(+)}+\widehat{x}_{(-)}\right) \bar{x}+\widehat{x}_{(+)} \widehat{x}_{(-)} \leq 0,
$$

where $\bar{x} \equiv q^{n}-\bar{q}$ and $\widehat{x}_{( \pm)} \equiv x_{( \pm)}(\widehat{\boldsymbol{\kappa}})$.

Since the left hand side of (C14) is a quadratic function of $\bar{x},(\mathrm{C} 14)$ can be satisfied in the relevant range in which $\bar{x}>0$ only if:

$$
\left(\widehat{x}_{(+)}+\widehat{x}_{(-)}\right)^{2} \geq 4(1+\xi(\widehat{\tau})) \widehat{x}_{(+)} \widehat{x}_{(-)} .
$$

Using (C11), (C8) and (B3), the above condition can be written as:

$$
1 \geq \frac{(1+\xi(\widehat{\tau}))\left(1-\widehat{\delta}_{\min }\right) F^{e}}{\Pi^{m}}=\frac{16 n}{(n+1)^{2}} \widehat{\tau}(1+\xi(\widehat{\tau})) .
$$

Define $T_{n}$ as the solution to the following equation:

$$
1=\frac{16 n}{(n+1)^{2}} T_{n}\left(1+\xi\left(T_{n}\right)\right)
$$

Since $\xi^{\prime}(\tau)>0$, then $T_{n}$ is unique and strictly positive. Using this, (C15) can be stated as $\widehat{\tau} \leq T_{n}$. Substituting (C11) into this condition and rearranging the terms yields $F^{e} \leq \widehat{F}$ where $\widehat{F}$ is given by:

$$
\widehat{F} \equiv \frac{16 n T_{n}}{(n+1)^{2}}\left(1+\frac{2(n+1) \Delta}{(n-1) q^{n}}\right) \Pi^{m} .
$$

The analysis thus implies that, for any $\delta \in \widehat{\Omega}_{F}$, (C10) can be satisfied only if $F^{e} \leq \widehat{F}$. In addition, it also implies that, for $F^{e}=\widehat{F}$ and $\delta=\widehat{\delta}_{\min }$, there exists a unique $(\widehat{\alpha}, \widehat{q})$ such that:

$$
\Psi\left(\widehat{\alpha}, \widehat{q}, \widehat{\delta}_{\min }, \widehat{F}, \Delta, n\right)=0
$$


By performing routine calculations, one can verify:

$$
\begin{aligned}
\widehat{q} & =q^{n}\left(1-\frac{8(n-1) T_{n}}{(n+1)^{2}}\right) \\
\widehat{\alpha} & =\sqrt{\frac{T_{n}}{1+T_{n}}} \frac{b(\widehat{q}, \widehat{\boldsymbol{\kappa}})}{\widehat{\delta}_{\min } \widehat{F}}=\sqrt{\frac{T_{n}}{1+T_{n}}} \frac{16 n T_{n}}{(n+1)^{2}} \frac{(n-1) q^{n}}{2(n+1) \Delta} .
\end{aligned}
$$

Note that, for $(\widehat{\alpha}, \widehat{q})$ to be sustainable in equilibrium, it must also satisfy:

$$
\Phi\left(\widehat{\alpha}, \widehat{q}, \widehat{\delta}_{\min }, \widehat{F}, n\right) \leq 0
$$

Since $\Psi(\cdot)$ and $\Phi(\cdot)$ are monotonic functions of $F^{e}$ (i.e., $\partial \Psi / \partial F^{e}>0$ and $\partial \Phi / \partial F^{e}>$ 0 ), it follows that $\bar{F}=\widehat{F}$ if (C20) is satisfied, and $\bar{F}<\widehat{F}$ otherwise.

Note that (C19), in particular, implies that $\widehat{\alpha}$ approaches infinity, as $\Delta$ tends to zero. In which case, (C20) cannot be satisfied because $g(\cdot)$ is bounded from above. Thus, it must necessarily be $\bar{F}<\widehat{F}$ for $\Delta$ small enough. The following lemma states the conditions under which $\bar{F}=\widehat{F}$.

Lemma C1. (C20) is satisfied for $\Delta$ large enough and $n \leq 12$.

Proof. Since $\widehat{\delta}_{\min }, \widehat{F}$ and $\widehat{\alpha}$ are continuous functions of $\Delta$ and $n$ (as implied by (B3), (C17) and (C19), respectively), then the composite function $\Phi\left(\widehat{\alpha}, \widehat{q}, \widehat{\delta}_{\min }, \widehat{F}, n\right)$ is continuous with respect to these variables. Thus, it suffices to show that (C20) can be satisfied for the maximal value of $\Delta$ which is equal to $q^{n}$ here. In which case, by continuity, it will also be satisfied for $\Delta$ 's sufficiently close to $q^{n}$.

Substituting $\Delta=q^{n}$ into (C19) yields:

$$
\left.\widehat{\alpha}\right|_{\Delta=q^{n}}=\sqrt{\frac{T_{n}}{1+T_{n}}} \frac{16 n T_{n}}{(n+1)^{2}} \frac{(n-1)}{2(n+1)} .
$$

Note that $(\mathrm{C} 16)$, taken with the fact that $\xi(\cdot) \geq 0$, implies that $16 n T_{n} /(n+1)^{2}<1$ and, therefore, $\left.\widehat{\alpha}\right|_{\Delta=q^{n}}<1$.

Using (B3), (C1), (C17) and (C18), one can verify:

$$
\left.\frac{g\left(\widehat{q}, \widehat{\delta}_{\min }, n\right)}{\widehat{F}}\right|_{\Delta=q^{n}}=\frac{2(n-1)}{(3 n+1)}\left(1-\frac{\left(n^{2}+8 n-1\right) T_{n}}{(n+1)^{2}}\right),
$$

which is lower than one.

Substituting (C21) and (C22) into the expression for $\Phi(\cdot)$ and rearranging the terms 
yields:

$$
\begin{aligned}
& \left.\Phi\left(\widehat{\alpha}, \widehat{q}, \widehat{\delta}_{\min }, \widehat{F}, n\right)\right|_{\Delta=q^{n}} \\
= & \frac{2(n-1)}{(3 n+1)}\left[\frac{16 n T_{n}}{(n+1)^{2}}\left(\sqrt{\frac{T_{n}}{1+T_{n}}} \frac{3 n+1}{4(n+1)}+\frac{n^{2}+8 n-1}{16 n}\right)-1\right] .
\end{aligned}
$$

Using the above condition, (C20) evaluated at $\Delta=q^{n}$ takes the form:

$$
\frac{16 n T_{n}}{(n+1)^{2}}\left(\sqrt{\frac{T_{n}}{1+T_{n}}} \frac{3 n+1}{4(n+1)}+\frac{n^{2}+8 n-1}{16 n}\right) \leq 1 .
$$

Since $\xi(\tau)$ is an increasing function while the fraction $16 n /(n+1)^{2}$ decreases as $n$ increases, then $T_{n}$ (defined by (C16)) must be an increasing function of $n$. From the condition $\left.\tau(1+\xi(\tau))\right|_{\tau=1 / 8}<(n+1)^{2} /\left.16 n\right|_{n=2}$ it follows that $T_{2}>1 / 8$. As a result, $T_{n} \geq T_{2}>1 / 8$ for all $n \geq 2$.

Next, since $\xi^{\prime}(\tau)>0$ and $\left.\xi(\tau)\right|_{\tau=1 / 8}=1$, then $\xi(\tau)>1$ for all $\tau>1 / 8$. Taken together, this condition and $T_{n}>1 / 8$ imply that $T_{n}\left(1+\xi\left(T_{n}\right)\right)>2 T_{n}$. In turn, this condition and the fact that $T_{n}$ satisfies (C16) imply that $16 n T_{n} /(n+1)^{2}<1 / 2$. Finally, using the latter condition and $T_{n} /\left(1+T_{n}\right)<1$, we have:

$$
\begin{aligned}
\varphi(n) & \equiv \frac{1}{2}\left(\frac{3 n+1}{4(n+1)}+\frac{n^{2}+8 n-1}{16 n}\right) \\
& >\frac{16 n T_{n}}{(n+1)^{2}}\left(\sqrt{\frac{T_{n}}{1+T_{n}}} \frac{3 n+1}{4(n+1)}+\frac{n^{2}+8 n-1}{16 n}\right) .
\end{aligned}
$$

Since $\varphi(n)$ is an increasing function and $\varphi(12) \simeq 0.98,(\mathrm{C} 23)$ is satisfied for all $n \leq 12$.

Lemma C2. $\widehat{F} \geq \Pi^{m}$ for $\Delta$ large enough and $n \leq 12$.

Proof. It suffices to show that $\widehat{F}$ evaluated at the the maximal value of $\Delta$ (which is equal to $q^{n}$ ) is strictly larger than $\Pi^{m}$, provided that $n \leq 12$. In which case, by continuity, this will also hold true for $\Delta$ 's sufficiently close to $q^{n}$.

Substituting $\Delta=q^{n}$ into (C17) yields:

$$
\left.\frac{1}{\Pi^{m}} \widehat{F}\right|_{\Delta=q^{n}}=\frac{16 n T_{n}}{(n+1)^{2}} \frac{(3 n+1)}{(n-1)} .
$$

Note that $\tau(1+\xi(\tau))<4 \tau(\tau+1 / 2)$ for all $\tau>0$. Taken together, this condition and (C16) imply that $(n+1)^{2} / 16 n<4 T_{n}\left(T_{n}+1 / 2\right)$. From the latter condition it 
follows:

$$
T_{n}>\frac{1}{4}\left(\sqrt{1+\frac{(n+1)^{2}}{4 n}}-1\right)=\frac{(n+1)^{2}}{16 n}\left(\sqrt{1+\frac{(n+1)^{2}}{4 n}}+1\right)^{-1} .
$$

Using (C24) and (C25), we have:

$$
\left.\frac{1}{\Pi^{m}} \widehat{F}\right|_{\Delta=q^{n}}>\psi(n) \equiv \frac{3 n+1}{n-1}\left(\sqrt{1+\frac{(n+1)^{2}}{4 n}}+1\right)^{-1} .
$$

Since $\psi(n)$ is a decreasing function and $\psi(12) \simeq 1.08$, the conclusion follows.

\section{Proof of Proposition 5}

The proof proceeds by way of two lemmas.

Lemma D1. Explicit collusion cannot be sustained as $\delta$ tends to zero.

Proof. Any sustainable outcome $(\bar{\alpha}, \bar{q})$ must satisfy (OD) and (HD). Consider first (OD). Since the function $\Pi^{e}(\bar{q})-\bar{\alpha} F^{e}$ is bounded from above, the left hand side of (OD) must approach zero, as $\delta$ tends to zero, which is possible only if $\bar{q}$ approaches $q^{n}$.

Consider now (HD). First, let us show that the left hand side of (HD) must be strictly positive as $\delta$ tends to zero. Suppose, to the contrary, that it is not. In which case, for $\delta$ small enough (HD) boils down to the following two conditions:

$$
\begin{aligned}
\max _{z \geq 0}\left((n+1)\left(q^{n}-\bar{q}\right)-\right. & \left.\frac{\delta}{1-\delta} \frac{\Pi^{e}(\bar{q})-\bar{\alpha} \delta F^{e}}{2 \Delta}-z\right) z=0 \\
\bar{\alpha}\left(\Pi^{e}(\bar{q})-(1-\delta+\bar{\alpha} \delta) F^{e}\right) & \geq 0 .
\end{aligned}
$$

Since $\Pi^{e}\left(q^{n}\right)=0, \partial \Pi^{e}\left(q^{n}\right) / \partial q<0$ and $\bar{\alpha}>0$, (D2) can be satisfied only if $\bar{q}<q^{n}$. In turn, (D1) can be satisfied only if the slope of the maximand in (D1) evaluated at $z=0$ is non-positive. This yields (using (C6)):

$$
\begin{aligned}
\bar{\alpha} \delta F^{e} & \leq \Pi^{e}(q)-\frac{2 \Delta(1-\delta)}{\delta}(n+1)\left(q^{n}-q\right)=-b(\bar{q}, \boldsymbol{\kappa}) \\
& =-\left(q^{n}-\bar{q}\right)\left(n\left(q^{n}-\bar{q}\right)+2 \Delta(n+1) \frac{\widehat{\delta}_{\min }-\delta}{\widehat{\delta}_{\min } \delta}\right) .
\end{aligned}
$$

The above condition necessarily implies that $\bar{q}>q^{n}$ for all $\delta<\widehat{\delta}_{\min }$. Thus, (D1) and (D2) contradict each other. 
In the proof of Proposition 4 it was established that, for a given $\kappa=\left(\delta, F^{e}, \Delta, n\right)$, (ND) can be satisfied only if:

$$
d(\bar{q}, \boldsymbol{\kappa}) \geq \xi(\tau(\boldsymbol{\kappa})) b(\bar{q}, \boldsymbol{\kappa}),
$$

where $\xi(\tau)=2 \tau(1+\sqrt{1+1 / \tau})$ while $b(\bar{q}, \boldsymbol{\kappa}), d(\bar{q}, \boldsymbol{\kappa})$ and $\tau(\boldsymbol{\kappa})$ are given by (C3), (C4) and (C5), respectively. Substituting (C6) and (C7) into (D3) yields (using that $\left.\bar{x}=q^{n}-\bar{q}\right)$ :

$$
n\left(\bar{x}-x_{(-)}(\boldsymbol{\kappa})\right)\left(x_{(+)}(\boldsymbol{\kappa})-\bar{x}\right) \geq \bar{x} \xi(\tau(\boldsymbol{\kappa}))\left(n \bar{x}+2 \Delta(n+1) \frac{\widehat{\delta}_{\min }-\delta}{\widehat{\delta}_{\min } \delta}\right),
$$

where $x_{( \pm)}(\boldsymbol{\kappa})$ is given by $(\mathrm{C} 8)$. By rearranging the terms and dividing by $n$, the above condition writes as:

$$
\zeta_{2}(\boldsymbol{\kappa}) \bar{x}^{2}-\zeta_{1}(\boldsymbol{\kappa}) \bar{x}+\zeta_{0}(\boldsymbol{\kappa}) \leq 0 .
$$

where $\zeta_{2}(\boldsymbol{\kappa}) \equiv 1+\xi(\tau(\boldsymbol{\kappa})), \zeta_{0}(\boldsymbol{\kappa}) \equiv x_{(+)}(\boldsymbol{\kappa}) x_{(-)}(\boldsymbol{\kappa})$ and

$$
\zeta_{1}(\boldsymbol{\kappa}) \equiv x_{(+)}(\boldsymbol{\kappa})+x_{(-)}(\boldsymbol{\kappa})-\frac{2 \Delta(n+1)}{n} \frac{\widehat{\delta}_{\min }-\delta}{\widehat{\delta}_{\min } \delta} \xi(\tau(\boldsymbol{\kappa})) .
$$

Using (B3), (C5) and (C8), one can verify:

$$
\begin{aligned}
\lim _{\delta \rightarrow 0} \zeta_{2}(\boldsymbol{\kappa}) & =1 \\
\lim _{\delta \rightarrow 0} \zeta_{1}(\boldsymbol{\kappa}) & =\left(\frac{n-1}{n} q^{n}\right)\left(1-\frac{n+1}{2} \sqrt{\frac{F^{e}}{n \Pi^{m}}}\right), \\
\lim _{\delta \rightarrow 0} \zeta_{0}(\boldsymbol{\kappa}) & =\left(\frac{n-1}{2 n} q^{n}\right)^{2} \frac{F^{e}}{\Pi^{m}} .
\end{aligned}
$$

Taken together, the above conditions imply that for $\delta$ small enough (D4) approximates to:

$$
\bar{x}^{2}-\left(\frac{n-1}{n} q^{n}\right)\left(1-\frac{n+1}{2} \sqrt{\frac{F^{e}}{n \Pi^{m}}}\right) \bar{x}+\left(\frac{n-1}{2 n} q^{n}\right)^{2} \frac{F^{e}}{\Pi^{m}}+O(\delta) \leq 0,
$$

which cannot be satisfied for $\bar{x}$ close enough to zero.

Thus, explicit collusion is not sustainable for $\delta$ small enough. On the other hand, it must be sustainable for $\delta$ large enough (since in this case tacit collusion is sustainable and, by Proposition 4, so is explicit one). Taken with the fact that (OD) and (HD) are monotonic in $\delta$, this implies that there must exist a threshold $\bar{\delta}_{\min } \in\left(0, \widehat{\delta}_{\min }\right]$ such 
that explicit collusion is sustainable if and only if $\delta \geq \bar{\delta}_{\text {min }}$.

Lemma D2. If $F^{e}<\bar{F}$, then $\bar{\delta}_{\min }<\widehat{\delta}_{\min }$.

Proof. From Proposition 3 it follows that $\left(\mathrm{HD}^{\mathrm{tc}}\right)$ is binding for $\delta=\widehat{\delta}_{\text {min }}$. By Proposition 4 , there always exists $(\bar{\alpha}, \bar{q})$ satisfying (C9) and (C10) when $\delta=\widehat{\delta}_{\text {min }}$ and $F^{e}=\bar{F}$. Note that the left hand sides of (C9) and (C10) are continuous functions of $\delta$ and decreasing functions of $F^{e}$. Thus, for $F^{e}<\bar{F}$ there must exist $\delta_{F}>0$ such that for any $\delta \in\left(\widehat{\delta}_{\min }-\delta_{F}, \widehat{\delta}_{\min }\right)$ both constraints are satisfied for some $(\bar{\alpha}, \bar{q})$. 


\section{References}

Abreu, D., Pearce, D., Stachetti, E., (1986), "Optimal Cartel Equilibria with Imperfect Monitoring," Journal of Economic Theory 39, 251-269.

Abreu, D., Pearce, D., Stachetti, E., (1990), "Toward a Theory of Discounted Repeated Games with Imperfect Monitoring," Econometrica 58, 1041-1064.

Athey, S., Bagwell, K., (2001), "Optimal Collusion with Private Information," The RAND Journal of Economics 32, 428-465.

Ben-Porath, E., Kahneman, M., (1996), "Communication in Repeated Games with Private Monitoring," Journal of Economic Theory 70, 281-297.

Compte, O., (1998), "Communication in Repeated Games with Imperfect Private Monitoring," Econometrica 66, 597-626.

Davies, S., Olczak, M., (2008), "Tacit versus Overt Collusion Firm Asymmetries and Numbers: What's the Evidence?" CCP Working Paper 08-32.

Fudenberg, D., Levin, D., Maskin, E., (1994), "The Folk Theorem with Imperfect Public Information," Econometrica 62, 997-1039.

Genesove, D., Mullin, W., (2001), "Rules, Communication and Collusion: Narrative Evidence from the Sugar Institute Case," American Economic Review 91, 379-398.

Green, E., Porter, R., (1984), "Noncooperative Collusion under Imperfect Price Information," Econometrica 52, 87-100.

Harrington, J., (2006), "How Do Cartels Operate?" Foundations and Trends in Microeconomics 2 (1), 1-105.

Harrington, J., Skrzypacz, A., (2011), "Private Monitoring and Communication in Cartels: Explaining Recent Collusive Practices," Forthcoming in American Economic Review.

Hay, G., (1982), "Oligopoly, Shared Monopoly, and Antitrust Law," Cornell Law Review 67, 439-481.

Horner, J., Jamison, J., (2007), "Collusion with (Almost) No Information," The RAND Journal of Economics 38(3), 804-822.

Kandori, M., Matsushima, H., (1998), "Private Observation, Communication and Collusion," Econometrica 66, 627-652.

Kuhn, K.-U., (2001), "Fighting Collusion by Regulating Communication between Firms," Economic Policy 32, 1-37.

Levenstein M., Suslow, V., (2006), "Cartel Bargaining and Monitoring: The Role of Information Sharing," The Pros and Cons of Information Sharing, Mats Bergman, ed. 
Martin, S., (2006), "Competition Policy, Collusion, and Tacit Collusion," International Journal of Industrial Organization 24, 1299-1332.

McCutcheon, B., (1997), "Do Meetings in Smoke-Filled Rooms Facilitate Collusion?" Journal of Political Economy 105, 330-350.

Motta, M. (2004), Competition Policy: Theory and Practice, Cambridge University Press.

Porter, R., (1983), "Optimal Cartel Trigger Price Strategies," Journal of Economic Theory 29, 313-338.

Stigler, G., (1964), "A Theory of Oligopoly," Journal of Political Economy 77, $44-61$.

Turner, D., (1962), "The Definition of Agreement Under the Sherman Act: Conscious Parallelism and Refusal to Deal", Harvard Law Review 75(4), 655-706. 


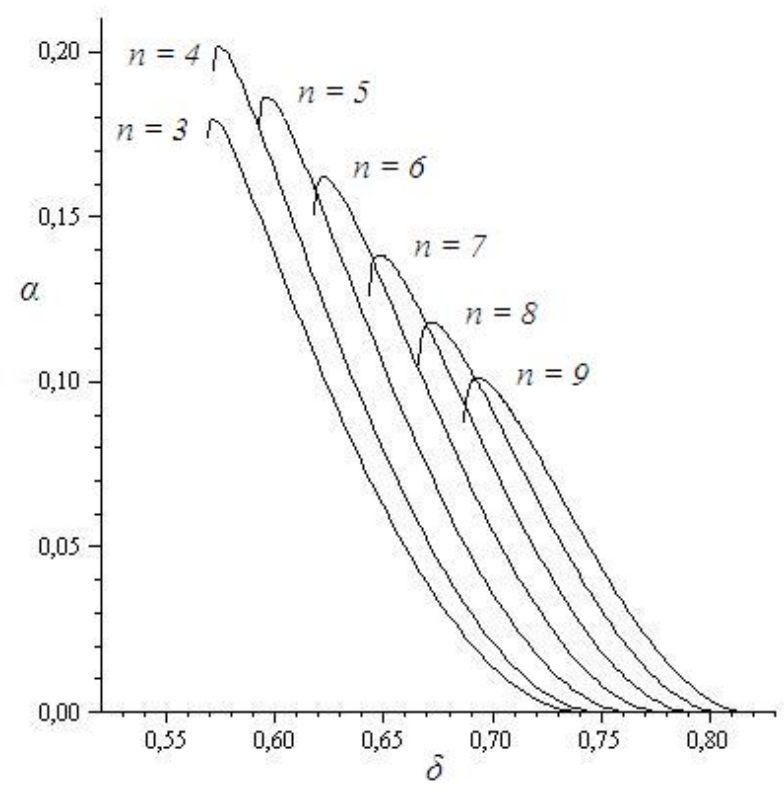

Figure 1: The probability $\alpha$ as a function of $\delta$ and $n$ given $\Delta=0.09$ and $F=0.02$.

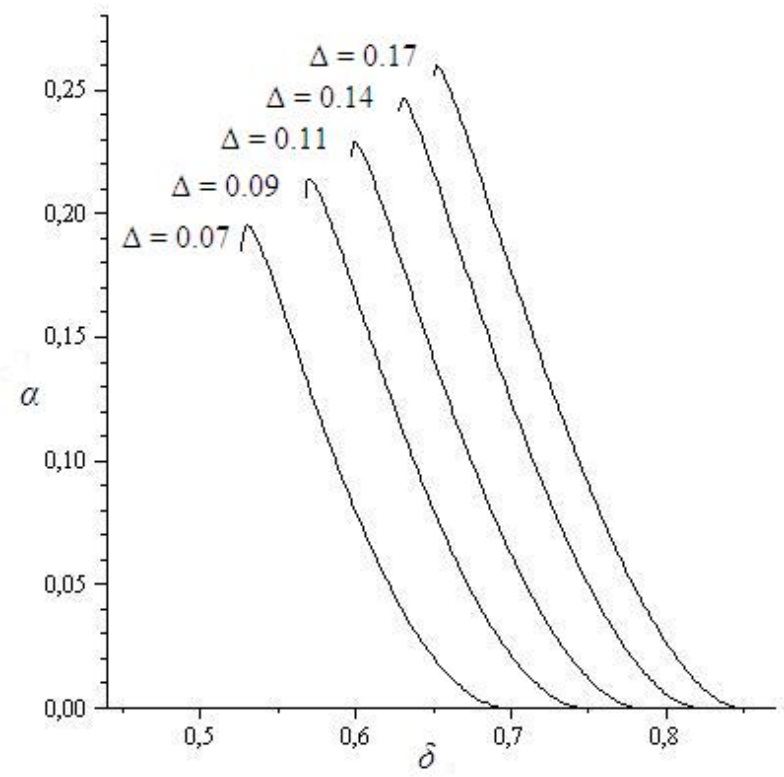

Figure 2: The probability $\alpha$ as a function of $\delta$ and $\Delta$ given $n=4$ and $F=0.02$. 